\title{
Orbifold transversality
}

\section{Michael McQuillan ${ }^{1}$}

Received: 6 June 2019 / Revised: 13 November 2020 / Accepted: 7 February 2021 /

Published online: 21 May 2021

(c) The Author(s) 2021

\begin{abstract}
We prove a best possible transversality theorem for maps from manifolds to orbifolds, and, more generally arbitrary differentiable Deligne-Mumford classifying champs, 0.1 , of groupoids $R \rightrightarrows U$ in separated, 0.2, manifolds. En passant, the essentially finite dimensional linear algebra nature of jet transversality is isolated.
\end{abstract}

Keywords Champ · Transversality

Mathematics Subject Classification 53C08 · 57N75

\section{Avant propos}

This is a paper in differential geometry employing a certain amount of category theory developed by Grothendieck in the context of algebraic geometry, and, unsurprisingly, different words are sometimes employed for the same idea according to the discipline, while, albeit less frequently, the same words can mean different things. As such, before the introduction properly said, let us clarify some terminology, which, invariably favours Grothendieck's choices wherein the natural sense of the word is closest to the idea, to wit:

0.1 Champ means what it means in [5, Exposé VI], i.e. stack in English. I'm told the intention of the translator (Mumford) was that stack should serve as an abbreviation of haystack, which is certainly the sense of champ (more accurately gerbe) in this context, but, in my experience, the abbreviation to stack is problematic because, independently, it has its own meaning and so creates a misleading idea.

0.2 Separated space, manifold, etc. means Hausdorff space, manifold, etc..

Michael McQuillan

mcquilla@mat.uniroma2.it

1 Università di Roma “Tor Vergata”, Via della Ricerca Scientifica 1, 00133 Rome, Italy 
0.3 Net map of spaces, manifolds, etc. means a local homeomorphism, or diffeomorphism according to the context, onto the image, i.e. the idea which is usually expressed in differential geometry written in English by the word immersed (and, as it happens, the translation of immersion as employed in EGA is embedding, which is another reason for preferring net).

0.4 Differentiable or infinitely differentiable if there is danger of confusion, manifold, orbifold, etc. means a smooth (as opposed to PL or whatever) manifold, orbifold, etc.. Since almost all the manifolds, orbifolds, etc. which occur will be infinitely differentiable, and the risk of confusion will be negligible, we'll often just say manifold, orbifold, etc., rather than (infinitely) differentiable manifold, orbifold, etc..

0.5 Smooth map, including the structure map to the final object is a map with smooth fibres, i.e. a submersion, so, tautologically a smooth space is one whose structure map is smooth. By definition all infinitely differentiable, 0.4 , manifolds, orbifolds, and even champs, 0.1 , have a smooth structure map. It is therefore a tautology to say smooth (infinitely) differentiable manifold, orbifold, etc.. Nevertheless, we'll often be making constructions whose essence is to identify fibres which are smooth, and whence, for emphasis, and uniquely for emphasis, we may say smooth (infinitely) differentiable manifolds, orbifolds, etc..

0.6 Étale map means a smooth map with discrete fibres, or, what is the same thing, a local diffeomorphism. As such, we're employing it in the sense of a local isomorphism in the category of interest, rather than the pre-Grothendieck sense of local homeomorphism. Consequently, the English distinction between local diffeomorphism and local homeomorphism is more precise, but "topology of local diffeomorphism" would have mean a mouthful, while, since the occurences are more frequent than the corresponding problem for net, 0.3, albeit Fact/Definition 2.4.(a) is always the cause, usages of local homeomorphism will be spelt out.

\section{Introduction}

Not unreasonably orbifold transversality is said to be false. Indeed if $\mathscr{O} \rightarrow \Sigma$ were an orbifold distinct from the underlying space $\Sigma$ then already for $\Sigma$ a Riemann surface and $E=\Omega_{\mathscr{O}}^{m}, m \geqslant 2$ copies of its co-tangent bundle, then although the zero section $[0] \hookrightarrow E$ is an embedded (smooth) sub-orbifold of co-dimension $2 m$, no matter the section $s$ of $E, s^{-1}[0]$, while it may be supported on a sub-orbifold of $\mathscr{O}$, will not only contain every point of the signature (and so be of co-dimension 2 rather than $2 m$ ) but if $z=0$ were a complex orbifold coordinate at a point where the signature is $n$ then the ideal of differentiable complex functions defined by $s^{-1}[0]$ is always, Fact A.1 with $a=1$, contained in $\left(z^{i} \bar{z}^{j} \mid j=1+i(n)\right)$. On the other hand, [8] or Remark 4.11, orbifolds have plenty of Morse functions, and the principle is that orbifold transversality valeat quantum potest valere, i.e. there is a certain local obstruction posed by whether some naturally defined vector bundle has sufficient sections, cf. 
Lemma 4.1-Lemma 4.2, to establish the said transversality locally, and, once this is true it follows, rather generally and wholly formally, globally.

Nevertheless, even in the absence of local obstructions, the formal aspect is not without its pitfalls, albeit, much less serious, since avoiding them is simply a matter of good formalism, i.e. a functorial use of language. For example, consider the basic case of transversality,

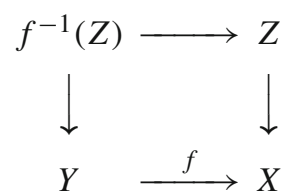

where $X, Y, Z$ are separated manifolds and $f$ is infinitely differentiable, then, for $Z \hookrightarrow X$ a sub-manifold, after a small perturbation of $f$, the fibre is either empty or a sub-manifold of $Y$ of the same co-dimension. Now, the right way to think of this is two fold, to wit:

(a) There is a property (P), e.g. closed embedding in the case in point, of maps between topological spaces which is stable under base change, i.e. if the right vertical in (1) has property (P) then the left vertical does too for any continuous map $f$.

(b) How to guarantee that the fibre in (1) is a (smooth) manifold, rather than just a topological space, and, preferably with the same co-dimension, which is a notion which has sense not just for embeddings but any net, 0.3, map?

Plainly, therefore, already for manifolds, we can pose slightly more general transversality problems by the simple expedient of replacing "closed embedding" by net together with any property, $(\mathrm{P})$, stable under base change.

At the same time, the unique way, Definition 2.6, consistent with the rest of mathematics, to ask that a map $\mathscr{Z} \rightarrow \mathscr{Y}$ of orbifolds, or better, differentiable champs, has a property $(\mathrm{P})$ is in terms of atlases and fibres. Consequently item (a) in the above schema will continue to be a tautology. As such the pitfall is not the formalism itself, but applying it. The example par excellence, which one may very well wish to use in practice to describe generic double points, and which is spelt out in Example/Definition/Warning 2.8, and Example A.8 et seq., is the diagonal map,

$$
\mathscr{Y} \stackrel{\Delta}{\rightarrow} \mathscr{Y} \times \mathscr{Y}
$$

which, already for $\mathscr{Y}$ an orbifold, and more generally a differentiable champs, is a closed embedding iff $\mathscr{Y}$ is a separated manifold, Example/Definition/Warning 2.8. As such, in general, the properties stable under base change for an orbifold, with underlying space separated, which one can assign to the diagonal, (2), are, net, and proper. Related to this is both the need to work with, and the naturality, of differentiable champs for posing transversality questions. Indeed, already for orbifolds, the locus $\mathscr{Z} \hookrightarrow \mathscr{Y}$ where an orbifold fails to be a manifold is very often an embedded (smooth) differentiable sub-champs but it is never a sub-orbifold, (13), and plainly even if one were only interested in orbifolds, transversality to $\mathscr{Z}$ is, invariably, of importance.

Concentrating, therefore, on the pleasing, since it has no local obstruction, case of maps from separated manifolds to differentiable champs we have, 
Proposition 1.1 (Proposition 4.5, Extra Fact 4.8-Extra Fact 4.10) Let $f: X \rightarrow \mathscr{Y}$ be a map from a separated manifold to a differentiable champ admitting an exponential map, Definition 3.8, from a neighbourhood of the zero section of the pull-back of the tangent space, e.g. the classifying champ of a groupoid $R \rightrightarrows U$ in separated manifolds, Proposition 1.2, so, inter alia no separability condition on $\mathscr{Y}$, and $m \in \mathbb{Z}_{>0}, r \in \mathbb{Z} \geqslant 0$ then for any closed subspace $V$ of $\mathrm{H}^{0}\left(X, f^{*} T_{\mathscr{Y}}\right.$ ) (in its usual Fréchet topology if $X$ is compact, and slightly adjusted, Set Up 4.9 \& Set Up 4.4, otherwise) separating $m, r$ multi-jets there is a residual subset, $K$, of an open ball about $0 \in V$ parametrising (via the exponential) deformations $g$ of $f$ such that if $\mathscr{Z} \rightarrow J_{r}^{m}(X, \mathscr{Y})$ is a representable separated net map from a (smooth) differentiable champ of co-dimension $q$ to the space of multi-jets, Sect. 3, enjoying a property (P) stable under base change, e.g. closed embedding, proper, discrete fibres, etc., then for $g \in K$, the left hand vertical of the fibre square,

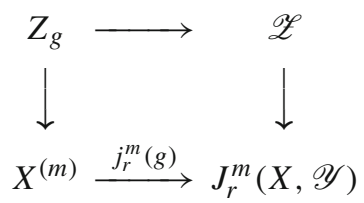

is empty or a net map from a separated manifold of co-dimension q enjoying property $(\mathrm{P})$, whence, in particular, empty if $q$ exceeds the dimension of $X$.

Here a champ in differentiable manifolds is exactly as in [5, Exposé VI], i.e. the 2sheaf, (7) et seq., associated to the action $R \rightrightarrows U$ of a groupoid in (not necessarily separated) manifolds, with étale, 0.6, source and sink, (7), albeit manifold is to be understood in the sense of the more general notion of B-manifold, Revision 2.2. As such, the condition that the right vertical in (3) is separated is non-empty. It is however unlikely to fail in practice, e.g. any representable map in separated manifolds is separated, Definition 2.6, and even then, its only role, Remark 4.6, is to permit the intervention of Sard's theorem, so, although it's a mild condition, cf. Remark 2.3, it is (if one is happy with a fibre which is a possibly non-separated manifold in the text book sense, Fact/Definition 2.4.(a)) overkill. In any case, and plainly, whenever $\operatorname{Hom}(X, \mathscr{Y})$ is known to exist, e.g., [15], under the hypothesis of Proposition 1.2 (or even just as a corollary of it) and the topology has a countable basis, the deformations $g \in \operatorname{Hom}(X, \mathscr{Y})$ satisfying the transversality statement Proposition 1.1 are residual, i.e. a countable intersection of open dense sets.

As such, the only substantive hypothesis of the theorem on $\mathscr{Y}$ is that it should admit an exponential map, Definition 3.8. Certainly, therefore, for identically the same reason which guarantees the usual exponential on manifolds the existence of a Riemann metric on $\mathscr{Y}$ would suffice. However, in the test case of the classifying champ of an arbitrary action,

$$
R=U \times \Gamma \rightrightarrows U
$$

by a discrete group on a manifold this means a $\Gamma$ invariant Riemannian metric, which, amongst all group actions, e.g. already on the sphere, is a rather strong condition, whereas an exponential map Definition 3.8, exists in considerable generality, to wit:

Proposition 1.2 (Fact 3.9) Let $f: X \rightarrow \mathscr{Y}$ be a map from a separated manifold to a differentiable champ, $\mathscr{Y}$, which is a classifying champ, (7), of the action of a groupoid 
$R \rightrightarrows U$ in separated manifolds, i.e. $U, R$ separated, with étale, 0.6, source and sink, then $f$ admits an exponential. In particular, therefore, the transversality statement Proposition 1.1 holds unconditionally for any map $f: X \rightarrow[U / R]$ when all of $X, U$ and $R$ are separated.

Now $R$ in (4) is separated iff $U$ is separated, so

Example 1.3 Suppose a discrete group $\Gamma$ acts on a separated manifold $Y$ (so in particular no separation condition on the topological quotient $Y / \Gamma$ ) then if $Z \hookrightarrow Y$ is a $\Gamma$-equivariant sub-manifold of co-dimension $q$ and $f: E \rightarrow Y$ a $\Gamma$-equivariant map from a $\Gamma$ torsor $E \rightarrow X$ over another separated manifold $X$ then for almost all $\Gamma$ equivariant deformations $g$ of $f$, i.e. all in some residual subset, $g^{-1}(Z)$ is empty or the fibre of $E$ over a co-dimension $q$ sub-manifold of $X$. Thus the only real caveat in this example is that the more complicated the action the less likely that there are non-trivial sub-objects, $Z$, to which it applies.

The paper is organised by way of a brief tour, Sect. 2, of the differential geometric manifestation of the general categorical considerations of [5] and [6]. Its contents while entirely well-known to experts, e.g. [1] or [7], are rarely employed in differentiable geometry. As such, its primary purpose is to explain, cf. Fact/Definition 2.4, Fact/Definition 2.7, and Example/Definition/Warning 2.8, why the language of [5, Exposé VI] is a necessity even if all one wants to talk about is transversality for orbifolds. The next section, Sect. 3, discusses the difference between jets in the sense of Grothendieck versus the sense of Thom, and culminates in the proof of Proposition 1.2. The final section, Sect. 4, applies this to the aforesaid transversality Proposition 1.1 by way of Thom's incidence correspondence, which is the type of thing one encounters more often in algebraic rather than differential geometry. As such it seemed appropriate to emphasise that when the subspace $V$ of Proposition 1.1 is finite dimensional (or an inverse limit thereof, Set Up 4.4, if $X$ isn't compact) the proof is straight out of the algebraic geometry playbook, albeit "generic smoothness" as encountered algebraically gets replaced by Sard's theorem, so that as soon as $V$ is infinite dimensional one has to use Smale's residual set formulation, Scholion 4.7, which, following, [16, 1.2 et seq.], we prefer to call almost all. All of which, at the eminently reasonable suggestion of the referee, is supplemented by an appendix, Sect. A, illustrating the properties of 1-dimensional champ such as $\mathbb{R} / \mathbb{Q}$, along with some examples of the main theorem. In particular, the very limited, if any, contribution of this note is the functoriality of the discussion about jets and, perhaps, a little cleaning of the role of the exponential map and the resulting linear analysis which, even in the infinite dimensional case, is little more, Scholion 4.7.(a)-(e), than finite dimensional linear algebra. Furthermore, no attempt is made to formulate Proposition 1.1 for maps from a champ/orbifold rather than a manifold even though it has already merited notable attention (albeit with the emphasis on PL rather than infinitely differentiable) in the specific case, e.g. [14] \& [12], of equivariant transversality since, as we've already shown by our initial example, short of an unrealistic hypothesis such as the absence of local obstructions what one can achieve is highly dependent on the local structure of what one might like to be transverse. 


\section{The need for precision}

Confusion about orbifold transversality tends to be the result of misuse of language. As such, we'll gather together some definitions from [5] \& [6] in the context of differentiable geometry and review perhaps the main source of confusion, Example/Definition/Warning 2.8, the diagonal map. To begin with we need a site, i.e.

Definition 2.1 By $\underline{B}$ is to be understood the category whose objects are disjoint unions of copies of open subsets of $\mathbb{R}^{n}$, with infinitely differentiable maps between them, where $n$ may vary throughout the union, which has a priori arbitrary cardinality, but in practice countable is invariably sufficient, and should be supposed if one prefers one's categories small. In any case the site $\underline{B}_{E}$ is $\underline{B}$ equipped with the étale topology, i.e. the topology on $\underline{B}$ generated, [6, II.1.3.1], by arrows of $\underline{B}$ which are étale, which, by the definition, 0.6 , of étale, is the same as that generated by disjoint unions of open embeddings by infinitely differentiable maps.

This quickly leads to the main protagonists,

Revision 2.2 A B-manifold, or just manifold if there is no danger of confusion, $X:=$ $U / R$, is the $\underline{\mathrm{B}}_{\mathrm{E} t}$ sheaf associated, [6, II.3], to the pre-sheaf,

$$
\underline{\mathrm{B}}^{\mathrm{op}} \rightarrow \underline{\text { Ens }}: T \mapsto U(T) / R(T)
$$

where

$$
R \underset{s}{\stackrel{t}{\rightrightarrows}} U
$$

is an equivalence relation in $\underline{\mathrm{B}}$ in which the source, $s$, and sink, $t$, are étale, and, as is usual, we confuse objects of $\underline{B}$ with their functors of points via the Yoneda embedding

$$
\underline{\mathrm{B}} \rightarrow \operatorname{Hom}\left(\underline{\mathrm{B}}^{\mathrm{op}}, \underline{\mathrm{Ens}}\right): V \mapsto h_{V}, \quad \text { where: } \quad h_{V}(T):=\operatorname{Hom}(T, V)=: V(T) \text {. }
$$

Consequently we have a site $\underline{\mathrm{B}}_{\mathrm{E}}^{\prime}{ }_{\mathrm{E}}$ where the underlying category $\underline{\mathrm{B}}^{\prime}$ has objects $\underline{\mathrm{B}}-$ manifolds with infinitely differentiable maps between them (see Definition 2.6 below), and the topology is that generated by surjective étale maps (again see Definition 2.6). In particular if (6) were only a groupoid in $\underline{B}$, or even more generally, if probably not in practice, $\underline{\mathrm{B}}^{\prime}$, cf. Remark 2.3, with étale source and sink, a (differentiable) champ, $\mathscr{X}:=[U / R]$, is the 2 sheaf associated, $[11,3.2]$, to the 2 pre-sheaf

$$
\underline{\mathrm{B}} \rightarrow \underline{\mathrm{Grpd}}: T \mapsto(R(T) \rightrightarrows U(T))
$$

where Grpd is the 2-category of groupoids in sets. As such if by points of $[U / R]$ we understand the objects in $[U / R](\mathrm{pt})$ modulo isomorphism, then the space like points are those with trivial stabiliser.

Allowing the groupoid in (7) to belong to $\underline{B}^{\prime}$ rather than just $\underline{B}$ merits,

Remark 2.3 Restricting (7) to groupoids in $\underline{B}$ is more than adequate for any imaginable application, and is in the same vein as typical hypothesis in algebraic geometry for 
avoiding pathology such as separated diagonal, [11, 4.1]. Consequently, the principal reason for allowing it is to illustrate that there is almost no restriction, albeit cf. Remark 4.6 for further details, on the generality in which transversality holds.

In any case, plainly there are many more manifolds according to Revision 2.2 than those one finds in text books, so it's useful to observe

Fact/Definition 2.4 By definition a relation $R \underset{s}{\stackrel{t}{\rightrightarrows}} U$ in $\underline{\mathrm{B}}$, with étale source and sink,

(a) Defines a manifold in the text book sense if for $Q$ its quotient in the category of topological spaces, Top, $U \rightarrow Q$ is a local homeomorphism.

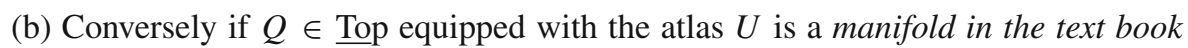
sense then the sub-sheaf of the restriction to $\underline{\mathrm{B}}$ of $\operatorname{Hom}_{\mathrm{Top}}(\bullet, Q)$ of differentiable mappings in the text book sense is the sheaf $U / R$ of Revision 2.2.

(c) Consequently, the category of differentiable manifolds in the text book sense is a full sub-category of manifolds in the sense of Revision 2.2.

(d) There are, however, more manifolds in the latter sense than there are in the text book sense, e.g. $\mathbb{R} / \mathbb{Q}$ is a manifold.

(e) Nevertheless if a $\underline{B}$-manifold is separated, i.e. $R \stackrel{s \times t}{\longrightarrow} U \times U$ is a closed embedding, then it is a manifold in the text book sense, i.e. belongs to the image of the aforesaid fully faithful functor, with, in addition $Q$ separated.

Proof Logically items (a) and (b) are definitions, so don't need proof. However, if one were to consult a text book on differentiable geometry one would find (a) and (b), so (c) is immediate, while $\mathbb{R} / \mathbb{Q}$ is clearly a B-manifold. If, however, this were a manifold in the text book sense then we'd have a fibre square in Top,

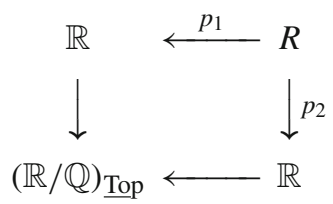

with the left vertical and bottom horizontal local homeomorphisms. Consequently all the arrows in (8) are local homeomorphisms. On the other hand, Top quotients qua naked sets are also Ens quotients, so there is a continuous set bijection,

$$
\coprod_{q \in \mathbb{Q}} \mathbb{R} \times q \rightarrow R
$$

whose composition with, say, $p_{1}$ is the supposed étale map $s$, and since $p_{1}$ is a local homeomorphism, (9) is also open, and whence a homeomorphism. At the same time $R$ is homeomorphic to

$$
\{(x, x+q) \mid x \in \mathbb{R}, q \in \mathbb{Q}\} \subset \mathbb{R} \times \mathbb{R}
$$


with the sub-space topology, so, if, for example, $q_{n} \rightarrow 0$ then $\left(0, q_{n}\right) \rightarrow(0,0)$ in $R$ from which the absurdity that this happens in the coproduct

$$
\coprod_{q \in \mathbb{Q}} \mathbb{R} \times q .
$$

Finally, by (a)-(c), separated manifolds in the text book sense are separated manifolds, so it remains to check the converse, i.e.

Claim 2.5 If $R \underset{s}{\stackrel{t}{\rightrightarrows}} U$ is a relation in $\underline{\mathrm{B}}$ with étale source and sink such that $R \stackrel{s \times t}{\longrightarrow}$ $U \times U$ is a closed embedding, then $U \rightarrow(U / R)_{\text {Top }}$ is a local homeomorphism and $(U / R)_{\text {Top }}$ is separated.

Proof of Claim 2.5 Fix $x \in U$ and a sequence of balls $B_{n} \ni x$ centred on it of radius collapsing to zero. On each ball there is an induced relation given by the fibre product,

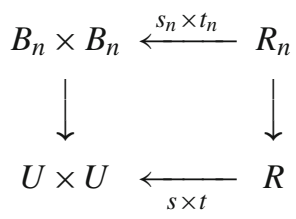

Now suppose for all $n, R_{n}$ contains a component other than the identity, i.e. there are non-identity arrows

$$
f_{n}: x_{n} \rightarrow y_{n} \in R_{n} .
$$

On the other hand the bottom, whence either, horizontal in (10) is a closed embedding, and $f_{n}$ converges to the identity $\mathrm{id}_{x}$. By the same token, or better because the identity map is continuous, the identities $\mathrm{id}_{x_{n}}$ of the source of (11) also converge to $\mathrm{id}_{x}$, and $s_{n}$ is étale, so eventually $f_{n}$ is the identity. Consequently, for $n \gg 0, R_{n} \rightrightarrows B_{n}$ is the identity relation, while by construction $\left(B_{n} / R_{n}\right)_{\text {Top }}$ embeds in $(U / R)_{\text {Top }}$, so eventually $B_{n} \rightarrow\left(B_{n} / R_{n}\right)_{\text {Top }}$ is a homeomorphism. Finally being a closed embedding is local in $\underline{\text { Top}}$, so the diagonal of $(U / R)_{\text {Top }}$ is a closed embedding iff $s \times t: R \rightarrow U \times U$ is.

which in turn completes the proof of Fact/Definition 2.4.

Plainly maps whether in our enlarged category of manifolds, or 2-category of differentiable champs, are just functors or 2-functors as appropriate. The pertinent question is, therefore when are such maps open, closed, étale, etc., or more generally

Definition 2.6 A property, $(\mathrm{P})$, of maps in $\underline{B}$ is said to be étale local if it is closed under base change and $U \rightarrow V$ in $\underline{\mathrm{B}}$ has property $(\mathrm{P})$ iff there is a surjective étale map $V^{\prime} \rightarrow V$ such that the base change $U^{\prime}:=U \times{ }_{V} V^{\prime} \rightarrow V$ has property (P). For such properties one says that a map of $\underline{B}$-manifolds or differentiable champs has property $(\mathrm{P})$ exactly as encountered in $[11,4.14]$ et seq. on replacing, mutatis mutandis, algébriques by differentiable. In particular it makes sense to say that $V \rightarrow \mathscr{Y}$ is an étale map from an element of $\underline{\mathrm{B}}$, and we say that $f: \mathscr{X} \rightarrow \mathscr{Y}$ is representable in a full sub-category, E, of B-manifolds (e.g. separated ones), if the fibre $f_{V}$ of $V \rightarrow \mathscr{Y}$ 
of any étale map from an object of $\underline{B}$ belongs to $\underline{E}$. Thus, for example, the diagonal map of a manifold, resp. champ, is representable in $\underline{B}$, resp. $\underline{B}^{\prime}$, by (6), resp. (7), and, more pertinently, $f$ has property $(\mathrm{P})$ iff every such fibre has. In particular if $\mathrm{E}$ is any sub-category of separated manifolds then any map representable in $\underline{\mathrm{E}}$ is, a fortiori, separated.

To apply this to maps and properties of orbifolds requires an analogue of Fact/ Definition 2.4.(e), i.e.

Fact/Definition 2.7 Orbifolds with underlying space separated are (by definition and up to 2-equivalence) classifying champs such that $s \times t: R \rightarrow U \times U$ is proper with the set of non-space like points nowhere dense. More precisely if $s \times t: R \rightarrow U \times U$ is proper, then for every $x \in U$ there is a basis of neighbourhoods $U \supset U_{x} \ni x$ such that the induced groupoid $R_{x} \rightrightarrows U_{x}$ (defined exactly as in (10)) is isomorphic to an (independent of $U_{x}$ ) $A_{x}:=\operatorname{Aut}_{R}(x)$ (which by properness is finite) action $A_{x} \times U_{x} \rightrightarrows U_{x}$, while the topological quotient $(U / R)_{\text {Top }}$ (or equivalently sheaf quotient $U / R$ albeit, in general, as a Top-sheaf in the topology, Top $p_{\text {Ét }}$, generated by local homeomorphisms rather than a $\mathrm{B}_{\mathrm{E}_{\mathrm{t}}}$-sheaf) is a separated space with a basis of open neighbourhoods $\left(U_{x} / A_{x}\right)_{\text {Top. }}$ In particular, the map $\mu:[U / R] \rightarrow(U / R)_{\text {Top }}$ is universal amongst maps of the classifier to topological spaces (or better Top ${ }_{\text {Ét }}$-sheaves) and $\mu$, resp. $(U / R)_{\underline{T o p}}$, is called the moduli map, respectively space.

Proof For $a \in A_{x}$ choose neighbourhoods $N^{a} \ni a$ such that the source affords a diffeomorphism, $s^{a}$, with some (independent of $a$ ) neighbourhood $N \ni x$. Now retake the notations of the proof of Claim 2.5, albeit with a groupoid rather than a relation, and suppose that $R_{n}$ isn't eventually contained in the union of the $N^{a}$. Consequently we'd have the arrows $f_{n}$ of (11) in the complement of the $N^{a}$ with source and sink converging to $x$. By hypothesis, however, $s \times t$ is proper, so the $f_{n}$ converge to some $a \in A_{x}$, whence the absurdity that $f_{n}$ is eventually equal to $\left(s^{a}\right)^{-1}\left(x_{n}\right) \in N^{a}$. As such for $n \gg 0, R_{n}$ decomposes as a topological sum,

$$
\coprod_{a \in A_{x}} R_{n}^{a}:=N^{a} \cap R_{n} .
$$

Similarly, we can, for some possibly large $n$, conclude from $s \times t$ proper, and the continuity of the groupoid structure:

$$
R_{n}^{a} \times_{s} R_{n}^{b} \rightarrow R_{n}^{a b}, \text { along with, }\left(R_{n}^{a}\right)^{-1} \subseteq R_{n}^{a^{-1}},
$$

so, in particular, the latter inclusion, by symmetry is actually an identity. Consequently if for $n \gg 0$, we define,

$$
U_{x}:=\bigcap_{a} s^{a}\left(R_{n}^{a}\right) \text { then from (12), } A_{x} \times U_{x}:(a, u) \mapsto t\left(\left(s^{a}\right)^{-1}(u)\right)
$$

defines an $A_{x}$-action on $U_{x}$ whose groupoid $A_{x} \times U_{x} \rightrightarrows U_{x}$ is exactly that induced by $R$ on $U_{x}$. There is, therefore, an open embedding,

$$
\left(U_{x} / A_{x}\right)_{\underline{\mathrm{Top}}} \hookrightarrow(U / R)_{\underline{\mathrm{Top}}}
$$


and the resulting open sets are plainly a basis of the topology of $(U / R)_{\text {Top. Better }}$ still, although $U \rightarrow(U / R)_{\text {Top }}$ may no longer be a local homeomorphism it is at least open, while the fibre of the diagonal of $(U / R)_{\text {Top }}$ is the closed set $(s \times t)(R)$, so the diagonal of $(U / R)_{\text {Top }}$ is closed. Finally since $(\bar{U} / R)_{\text {Top }}$ is a co-equaliser in Top, $\mu$ is universal by definition.

Thus, for example, it follows that the definition of an open, respectively closed, subchamp of a classifying champ $\mathscr{X}=[U / R]$ of an étale groupoid $R \rightrightarrows U$ is,

an open, respectively closed, embedding representable in $\underline{B}$, Definition 2.6,

which can, even in natural examples, easily fail as illustrated by

Example/Definition/Warning 2.8 A champ $\mathscr{X}$ is said to be separated if its diagonal is proper, equivalently, as encountered in Fact/Definition 2.7, $s \times t: R \rightarrow U \times U$ is proper. According to the rules for functorial assignation of properties to the map $\mathscr{X} \rightarrow$ pt of Definition 2.6 this is unquestionably an abus de language, and even a serious one. Nevertheless its usage is (perhaps regrettably) standard since the more functorial statement, consistent with Definition 2.6, that $\mathscr{X} \rightarrow$ pt is separated iff the diagonal embeds is equivalent to $\mathscr{X}$ a separated manifold. Worse, this abus de language has consequences. Indeed, consider first the non-separated manifold, $M=U / R$, where $U$ is the union of two pointed circles, $(S, *)$, and $R$ is the relation where by we identify them off the points. Consequently the circles in $U$ afford distinct maps $f_{i}: S \rightarrow M, 1 \leqslant i \leqslant 2$, and the fibre

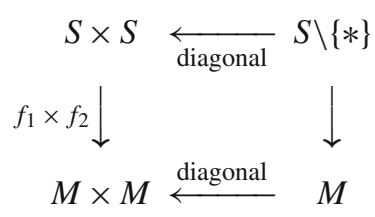

may be a manifold, but no amount of perturbing the $f_{i}$ will ever make it closed. Now while the problem here is that $M$ isn't separated this is per accidens rather than per se since if the diagonal in $M \times M$ were replaced by an honest closed sub-manifold in the sense of (13) the fibre in (14) after at worst a perturbation of the $f_{i}$ would be a closed sub-manifold. Now say a finite group $\Gamma$ acts on a separated manifold $X$ with fixed points, but not generically, e.g. the orientation reversing involution on the circle, then the classifier, $[X / \Gamma]$, of this action is an orbifold, and, or course, we have a fibre square,

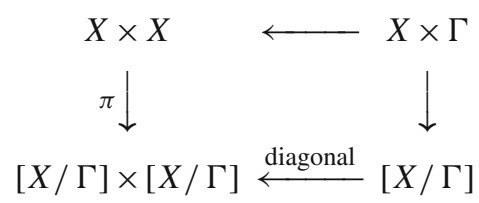

so that again, the fibre, although a manifold, is not a sub-manifold of $X \times X$ and no amount of perturbing $\pi$ will ever result in a better fibre than (15). Nevertheless, as before, if we were to replace the diagonal in (15) by an actual sub-manifold in the sense 
of (13), then, Proposition 4.5, the fibre, possibly after perturbation, is a sub-manifold of $X \times X$. In neither (14) nor (15), however, does the main theorem fail, but, rather its possible conclusions, which are spelt out in Example A.8, resp. Example A.9, are circumscribed by exactly the aforesaid optimality of the fibre in (14), resp. (15).

\section{Jets}

Google doesn't immediately reveal a reference for a functorial construction of Thom, rather than Grothendieck, jets. Nevertheless the latter may be used to define the former. Specifically if $X$ is a separated manifold there is an $r$ th thickening of the diagonal,

$$
X[r] \hookrightarrow X \times X
$$

which is a differentiable space in the sense of [13]. Specifically if $I_{\Delta}$ is the sheaf of ideals of infinitely differentiable functions, $\mathscr{A}_{X \times X}$, vanishing on $\Delta$ then $I_{\Delta}^{r+1}$ is closed in the (natural) Fréchet topology of infinitely differentiable functions, since, locally:

$$
f \in I_{\Delta}^{r+1} \text { iff } \partial_{1}^{a_{1}} \cdots \partial_{n}^{a_{n}}(f)=0, a_{1}+\cdots+a_{n} \leqslant r
$$

for a choice of vector fields $\partial_{i}$ normal to the diagonal. Similarly if $t: T \rightarrow X$ is a differentiable space over $X$, then the graph of $t$ has an $r$ th thickening, $T[r]$, defined via the fibre square,

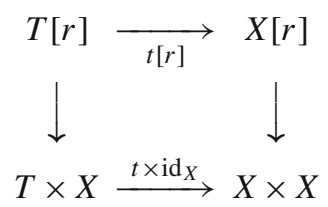

of differentiable spaces, and we observe,

Fact/Definition 3.1 For any separated (albeit, Fact/Definition 3.2, this is pro tempore and simply to avoid notational confusion by way of Fact/Definition 2.4.(e)), differentiable manifold $Y$ the Thom jets, $J_{r}(X, Y)$, [10, 2.4], represent the functor on $X$-differentiable spaces,

$$
T / X \mapsto \operatorname{Hom}_{X}(T[r], X \times Y)
$$

or, indeed, cf. (25), the functor (18) restricted to $\underline{B}$ provided the Hom set in (18) is understood in differentiable spaces, and, in any case, the structure of $T[r]$ as a $X$-space is given by the second projection in (17), so that (18) is equally,

$$
T / X \mapsto \operatorname{Hom}(T[r], Y)
$$

while the structure map (as an $X$-space) $p: J_{r}(X, Y) \rightarrow X$ is referred to as the source, and the (universal) evaluation map $q: J_{r}(X, Y) \rightarrow Y$ as the sink. In particular the Grothendieck jets, $\mathbb{V}\left(\left(\mathscr{P}_{X}^{r}\right)^{\vee}\right) \rightarrow X,[4,16.7]$, viewed (EGA convention of [3]) as a 
vector bundle over $X$ with sheaf of section $\mathscr{P}_{X}^{r}$ (rather than the infinitesimal space $X[r]$, whence this is the sheaf dual to the sheaf of differential operators of order $\geqslant-r$ ) are the Thom jets $J_{r}\left(X, \mathbb{G}_{a}\right)$.

Proof By definition, [10, p. 60], the fibre $J_{r}(X, Y)_{x}$ of the Thom jets over a point $x$ is the equivalence class, $[f]$, amongst local germs of maps $f$ from a neighbourhood of $x$ to $Y$ under the relation,

$$
f \sim g \text { iff } f^{*}=g^{*} \bmod \mathfrak{m}(x)^{r+1} .
$$

On the other hand if we have a map $F: T[r] \rightarrow Y$ then there is no local obstruction in lifting this to a map from $X$ to $Y$. In particular, if $t: T \rightarrow X$ were a point $x \in X$ then there is a lifting of $F$ to a germ $f$ of a map from an open neighbourhood of $x$ to $Y$, and two such liftings define the same $F$ iff they are related by (20). As such the functor (18) and the Thom jets have the same closed points, while a general $T$-point is just a smoothly varying family $F_{t}$ of jets of order $r$ which is precisely the (infinitely) differentiable structure on $J_{r}(X, Y)$ defined on [10, p. 61].

From which it follows,

Fact/Definition 3.2 Let $f: U \rightarrow U^{\prime}, g: V \rightarrow V^{\prime}$ be maps in $\underline{\mathrm{B}}$, then there are natural maps (more accurately natural transformations of functors)

$$
\begin{aligned}
& f^{*}: U_{f} \times_{U^{\prime}, p} J_{r}\left(U^{\prime}, V^{\prime}\right) \rightarrow J_{r}\left(U, V^{\prime}\right), \text { resp. } \\
& g_{*}: J_{r}\left(U^{\prime}, V\right) \rightarrow V_{g} \times_{U^{\prime}, q} J_{r}\left(U^{\prime}, V^{\prime}\right)
\end{aligned}
$$

which are isomorphisms if $f$, resp. $g$, is étale. In particular if both $f$ and $g$ are étale then we have a natural isomorphism,

$$
\left(f^{*}\right)^{-1} \times g_{*}: J_{r}(U, V) \stackrel{\sim}{\longrightarrow}(U \times V)_{f \times g} \times_{U^{\prime} \times V^{\prime}, p \times q} J_{r}\left(U^{\prime}, V^{\prime}\right) .
$$

Consequently if $X=U / A$, respectively $Y=V / B$, are $\underline{B}$-manifolds in the sense of (5) et seq. defined by relations with source and sink $p_{i}$, respectively $q_{i}, i=1$ or 2 , then (22) defines a relation

$$
J_{r}(A, B) \rightrightarrows J_{r}(U, V)
$$

with source $\left(p_{1}^{*}\right)^{-1} \times q_{1}$ and sink $\left(p_{2}^{*}\right)^{-1} \times q_{2}$ whose quotient (wholly compatibly with Fact/Definition 3.1 via Yoneda) is by definition the Thom jets $J_{r}(X, Y)$.

Proof That we have the maps (21) is just a diagram chase, while $f^{*}$, resp. $g_{*}$, is an isomorphism because the thickening of the graph (17), resp. an infinitesimal deformation, is unchanged by an étale map.

To profit from the formulation (18) observe that because their construction is étale local then in a way which is both analogous and simpler the Grothendieck jets $\mathscr{P}_{\mathscr{X}}^{r}$ continue to have perfect sense for a differentiable champ $\mathscr{X}$ wholly irrespectively of the fact that Example/Definition/Warning 2.8 prevents (16) et seq. from generalising as stated. As such, if $t: T \rightarrow \mathscr{X}$ is a map (from a space) then the $r$ th thickening, $T[r]$ 
of (17), of the graph of $t$ is given by a formula which does generalise, i.e. the ringed space,

$$
T[r]:=\left(T, t^{*} \mathscr{P}_{\mathscr{X}}^{r}\right)
$$

which in turn renders evident,

Fact/Definition 3.3 If $\mathscr{X}, \mathscr{Y}$ are differentiable champs (resp. separated) then for $T[r]$ as in (24), Thom jets define a differentiable champ (resp. separated) $J_{r}(\mathscr{X}, \mathscr{Y}) \rightarrow$ $\mathscr{X} \times \mathscr{Y}$, representing the 2-functor,

$$
T / \mathscr{X} \mapsto \operatorname{Hom}(T[r], \mathscr{Y}) \in \underline{\mathrm{Grpd}} \underline{\mathrm{d}}, \quad T \in \underline{\mathrm{B}} .
$$

More precisely if $[U / A]$, resp. $[V / B]$, are presentations of $\mathscr{X}$, resp. $\mathscr{Y}$, as classifiers of étale groupoids in $\underline{B}$-manifolds then $\mathscr{X} \times \mathscr{Y}$ is equivalent to the classifier $[U \times V / A \times B]$, while the sense of the Hom set in (25) is via the same 2-sheafication procedure prior to (7), albeit with the caveat, cf. (18), that the basic Hom sets on manifolds, e.g. (19), must be understood in differentiable spaces, so that just as in (23) the natural fibred squares of maps,

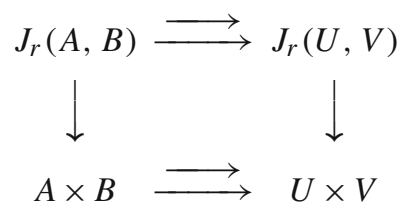

afforded by (21)-(22) define (top horizontals in (26)) an étale groupoid representing $J_{r}(\mathscr{X}, \mathscr{Y})$ mapping by the (jet) source and sink (verticals in (26) ) to $\mathscr{X} \times \mathscr{Y}$. Ultimately therefore, $J_{r}(\mathscr{X}, \mathscr{Y})$ is the classifier of a groupoid in B-manifolds, cf. Fact/Definition 3.1, which could have been defined in terms of charts and patching, so the infinitesimal formulation (25) results from the conceptual simplification that it affords.

Proof Since the isomorphisms (21) are actually natural transformations of functors there's nothing to do.

From which we have the tautology,

Definition 3.4 Let $f: \mathscr{X} \rightarrow \mathscr{Y}$ be a map then from the composition

$$
f[r]: \mathscr{X}[r] \rightarrow \mathscr{X} \times \mathscr{X} \stackrel{\operatorname{id} \times f}{\longrightarrow} \mathscr{X} \times \mathscr{Y}
$$

wherein the left hand side is understood as the classifying champ in differentiable spaces (i.e. replace $\underline{B}$ in Revision 2.2 by differentiable spaces) of the groupoid (in differentiable spaces with étale source and sink), implied by (24), i.e. $X[r]$ of (16) if $\mathscr{X}$ were the separated manifold $X$ of op. cit., then from the functorial definition (25), there is a map, the $r$ th jet,

$$
j_{r}(f): \mathscr{X} \rightarrow J_{r}(\mathscr{X}, \mathscr{Y})
$$


such that the diagram,

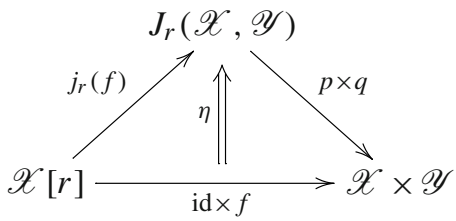

2-commutes by way of a unique natural transformation $\eta$.

In applying this in a relative fashion, i.e. just a smooth fibration $\mathscr{Y} \rightarrow \mathscr{X}$ rather than a product $\mathscr{Y} \times \mathscr{X}$, there is need for caution. For example, in respect of our immediate interest, vector bundles, an isomorphism between the two pull backs of $\mathscr{X}$ [2] $\rightrightarrows \mathscr{X}$ is a connection, which is necessarily non-canonical as soon as the bundle fails to be locally constant. As such, the correct definition is,

Definition 3.5 Let $\epsilon: E \rightarrow \mathscr{X}$ be a vector bundle, and $\left(p_{1}, p_{2}\right): \mathscr{X}[r] \rightarrow \mathscr{X}$ the projections, then the $r$ th space of Thom jets, $\epsilon_{r}: J_{r}(E) \rightarrow \mathscr{X}$, is the 2-functor,

$$
(T \stackrel{t}{\rightarrow} \mathscr{X}) \mapsto \operatorname{Hom}_{T[r]}\left(T[r], p_{2}^{*} E\right), \quad T \in \underline{\mathrm{B}}, \quad T[r] \text { as in }(24),
$$

which is, in fact, a vector bundle over $\mathscr{X}$. Indeed, if (cf. post (19) for our conventions) we identify $E$ with its sheaf of sections, i.e. $E=\mathbb{V}\left(\mathscr{E}^{\vee}\right)$, for a locally free sheaf $\mathscr{E}$ of $\mathscr{A}_{X}$ modules, then the right hand side of (28) is,

$$
\mathrm{H}^{0}\left(T[r], p_{2}^{*} \mathscr{E}\right)
$$

viewed as an $\mathscr{A}_{T}$ module by way of $p_{1}^{*}$, i.e. $\left(p_{1}\right)_{*} p_{2}^{*} \mathscr{E}$, and $\mathbb{V}\left(\left(\left(p_{1}\right)_{*} p_{2}^{*} \mathscr{E}\right)^{\vee}\right) \rightarrow \mathscr{X}$ (wherein duals don't commute with push-forward) represents (28). In particular, for any section, $s$, there is a tautological $r$-jet,

$$
j_{r}(s):=p_{2}^{*} s: \mathscr{X} \rightarrow J_{r}(E) .
$$

Before proceeding let us make a clarifying,

Remark 3.6 Modulo duality issues in the notation, the Thom jets with values in a vector bundle of 3.5 are bundle valued Grothendieck jets. Indeed for $\mathscr{E}$ a quasi-coherent sheaf the notation of $[4,16.7 .2 .1]$ is,

$$
\mathscr{P}_{\mathscr{X}}^{r}(\mathscr{E}):=\left(p_{1}\right)_{*} p_{2}^{*} \mathscr{E}
$$

while the notation for the jet map of (29) is, op. cit. 16.7.5.1, $d_{\mathscr{X}, \mathscr{E}}^{r}(s)$ or $d_{\mathscr{X}}^{r}(s)$. As such these definitions are not to be confused with $\mathscr{E} \otimes \mathscr{P}_{\mathscr{X}}^{r}$, or equivalently $\left(p_{1}\right)_{*} p_{1}^{*} \mathscr{E}$ the existence of a jet map for which, in the holomorphic case, is, already for $r=1$, globally obstructed by the Atiyah class.

The jets (27) and (29) may be related in the obvious way, 
Construction 3.7 Suppose a differentiable champ $\mathscr{Y}$ admits a Riemannian metric, e.g., but, cf. Fact/Definition 2.4.(d) \& Example/Definition/Warning 2.8, certainly not necessarily, $\mathscr{Y}$ separated $[8,5.4]$, then étale locally at a geometric point $y: \mathrm{pt} \rightarrow \mathscr{Y}$ there is an exponential map $\exp _{y}: B_{y} \rightarrow \mathscr{Y}$ from a sufficiently small ball $B_{y} \subset y^{*} T_{\mathscr{Y}}$, which is étale, but an embedding only if $\mathscr{Y}$ is a space at $y$. These glue together to a map

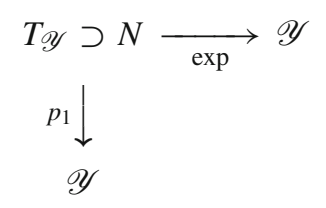

for a sufficiently small neighbourhood, $N$, of the zero section. As such if $f: \mathscr{X} \rightarrow \mathscr{Y}$ is given, then we equally have

$$
\begin{aligned}
& f^{*} T_{\mathscr{Y}} \supset f^{*} N \underset{\exp }{\longrightarrow} \mathscr{Y} \\
& p_{1} \downarrow \\
& \mathscr{X}
\end{aligned}
$$

wherein, as we've already indicated, $p_{1} \times$ exp always fails to be an embedding when $\mathscr{Y}$ is not a space, although it's always étale. In any case by way of the obvious variant,

$$
J_{r}\left(f^{*} N\right)=(T \stackrel{t}{\rightarrow} \mathscr{X}) \mapsto \operatorname{Hom}_{T[r]}\left(T[r], p_{2}^{*} f^{*} T_{\mathscr{Y}}\right)
$$

of (28), combined with (25) and (31), we have a map,

$$
j_{r}(\exp ): J_{r}\left(f^{*} N\right) \rightarrow J_{r}(\mathscr{X}, \mathscr{Y}) .
$$

Similarly if a section, $s$, of $f^{*} T_{\mathscr{Y}}$ takes values in $f^{*} N$, then we not only have $j^{r}(s)$ given by (29), but also $j_{r}(\exp (s))$ from (27), which, via another diagram chase, are related by,

$$
j_{r}(\exp (s))=j_{r}(\exp ) j_{r}(s)
$$

The linearisation of the study of deformations of $f$ implicit in (31) merits a definition which isolates the key point,

Definition 3.8 Let $f: \mathscr{X} \rightarrow \mathscr{Y}$ be a map of differentiable champs then we say that $f$ admits an exponential if there is an open neighbourhood $M$ of the zero section, [0], of $f^{*} T_{\mathscr{Y}}$ and a map,

$$
\begin{aligned}
& f^{*} T_{\mathscr{Y}} \supset M \underset{\exp }{\longrightarrow} \mathscr{Y} \\
& p_{1} \downarrow \\
& \mathscr{X}
\end{aligned}
$$

such that $f=\left.\exp \right|_{[0]}$ and $p_{1} \times \exp$ is étale. 
Now, as we've said, the existence of a Riemannian metric guarantees, Construction 3.7 , the existence of an exponential in the strong form (30)-(31), but much more generally,

Fact 3.9 Suppose a differentiable champ, $\mathscr{Y}$, is the classifying champ, (7), of a groupoid $R \rightrightarrows U$ with étale source and sink, $U \in \underline{\mathrm{B}}$ and $R$ a separated manifold then any map $f: X \rightarrow \mathscr{Y}$ from a separated manifold, Fact/Definition 2.4.(e), admits an exponential.

Proof Since $X$ is separated, there is a countable locally finite refinement,

$$
V:=\coprod_{\alpha \in A} V_{\alpha}
$$

of the cover $f^{-1} U$ in which not just every $V_{\alpha}$ is an (embedded) open subset of $X$ diffeomorphic to $\mathbb{R}^{n}$ but so too is every finite intersection of the $V_{\alpha}$ 's. In particular, therefore, we can identify $f$ with a functor,

$$
F: G:=V \times_{X} V=\coprod_{\alpha \beta} V_{\alpha \beta}\left(:=V_{\alpha} \cap V_{\beta}\right) \rightarrow R
$$

albeit we continue to use $f$ for the map on objects. In any case let,

$$
T_{0}:=\coprod_{\alpha} T_{\alpha}
$$

be an (eventually sufficiently small, whence variable) neighbourhood of the graph,

$$
\Gamma_{f}: V \stackrel{\mathrm{id} \times f}{\longrightarrow} V \times U
$$

and $T$ the connected components of the fibre,

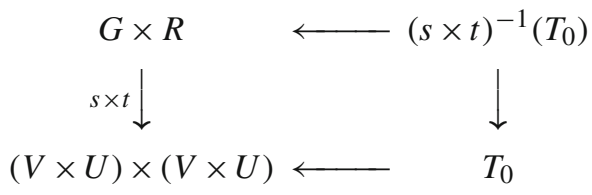

which contain the graph $\Gamma_{F}$ of $F$ of (35), where here, and elsewhere, we indiscriminantly employ $s, t$ for the source and sink of any groupoid that we may encounter, then we assert,

Claim 3.10 If the neighbourhood $T_{0}$ is sufficiently small then the source $s$, respectively the sink $t$ restricted to any connected component of $T$ is injective.

Proof of Claim 3.10 Fix a compact set,

$$
K=\coprod_{\alpha} K_{\alpha} \subset \subset V_{\alpha}
$$


so, inter alia, all but finitely many $K_{\alpha}$ empty, then it will suffice to prove that all sufficiently small neighbourhoods of $\Gamma_{f}(K)$ satisfy the proposition. As such, suppose that no neighbourhood of $\Gamma_{f}(K)$ satisfies the proposition, then, without loss of generality there are distinct sequences of arrows,

$$
v_{n} \times u_{n} \stackrel{a_{n} \times A_{n}}{\longrightarrow} v_{n}^{\prime} \times u_{n}^{\prime}, \quad v_{n} \times u_{n} \stackrel{b_{n} \times B_{n}}{\longrightarrow} v_{n}^{\prime \prime} \times u_{n}^{\prime \prime} \in V \times U
$$

belonging to a connected component of $T_{n}$ associated to a decreasing sequence of neighbourhoods $T_{0}(n)$, collapsing to the graph of $K$, according to the recipe of (37) et seq.. Now from the inclusions of connected open sets,

$$
V_{a} \hookleftarrow V_{\alpha \beta} \hookrightarrow V_{\beta}
$$

we have $v_{n}^{\prime}=v_{n}^{\prime \prime}$ in (39), while, after sub-sequencing, $v_{n}$, resp. $v_{n}^{\prime}$, converge to $v$, resp. $v^{\prime}$ in $K$. On the other hand $X$ is separated, so $a_{n}=b_{n}$ converges to $a \in G$, and both of $U$ and $R$ are separated so,

$$
u_{n} \rightarrow f(v), \quad u_{n}^{\prime}, u_{n}^{\prime \prime} \rightarrow f\left(v^{\prime}\right), \quad A_{n}, B_{n} \rightarrow F(a) .
$$

We can, however, take an open neighbourhood $\Omega \ni a \times F(a)$ on which $s$ is a homeomorphism, while for $n \gg 0$,

$$
v_{n} \times u_{n} \in s(\Omega), \quad A_{n}, B_{n} \in \Omega
$$

so, contrary to hypothesis, $A_{n}=B_{n}$ for $n \gg 0$.

In a similar vein we further assert,

Claim 3.11 Suppose the conclusion of Claim 3.10 and for any $\alpha, \beta \in A$, let $T_{\alpha \beta} \hookrightarrow T$ be the connected component of the graph of $V_{\alpha \beta}$ then it's also a connected component of the fibre,

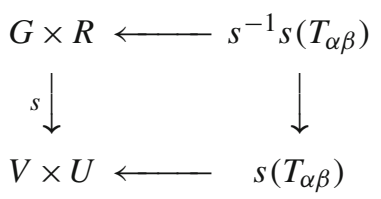

Proof of Claim 3.11 Say $x_{n} \in T_{\alpha \beta}$ converge to $x^{\prime}$ in the fibre of (40), then $s\left(x_{n}\right) \rightarrow$ $s\left(x^{\prime}\right)$. On the other hand, by definition, there is a $x \in T_{\alpha \beta}$ such that $s(x)=s\left(x^{\prime}\right)$, so under the hypothesis of Claim 3.10 and since $R$ is separated, $x_{n} \rightarrow x$, i.e. $T_{\alpha \beta}$ is closed in the fibre.

The final such assertion that we'll require is,

Claim 3.12 Again let $T_{0}$ satisfy the conclusion of Claim 3.10 then for any sufficiently small neighbourhood $T_{0}^{\prime}$ of the graph of $f$, (36), with $T^{\prime} \hookrightarrow G \times R$ associated to $T_{0}^{\prime}$ according to the recipe of (37) et seq. the image under composition of the composable arrows,

$$
T_{t}^{\prime} \times{ }_{s} T^{\prime}
$$


is contained in $T$, i.e. neighbourhood of (37) et seq. associated to the given $T_{0}$.

Proof of Claim 3.12 For each triple index the graph of the connected open $V_{\alpha \beta \gamma}=$ $V_{a} \cap V_{\beta} \cap V_{\gamma}$ affords a unique connected component,

$$
T_{\alpha \beta \gamma} \hookrightarrow T_{\alpha \beta t} \times{ }_{s} T_{\beta \gamma}
$$

which, by connectedness, must certainly map to $T_{\alpha \gamma}$. As such we need only take $T^{\prime}$ sufficiently small so that (41) is contained in the left hand side of (42). Similarly to the proof of Claim 3.10, with $K$ as in (38), suppose this is false for all open neighbourhoods of $K$, then there is a triple of indices $\alpha, \beta, \gamma$ and composable arrows,

$$
v_{n} \times u_{n} \stackrel{a_{n} \times A_{n}}{\longrightarrow} v_{n}^{\prime} \times u_{n}^{\prime} \stackrel{b_{n} \times B_{n}}{\longrightarrow} v_{n}^{\prime \prime} \times u_{n}^{\prime \prime}
$$

with $v_{n} \times u_{n}$, resp. $v_{n}^{\prime} \times u_{n}^{\prime}$, resp. $v_{n}^{\prime \prime} \times u_{n}^{\prime \prime}$, in increasingly small neighbourhoods of $K_{\alpha}, K_{\beta}, K_{\gamma}$ which fail to lie in $T_{\alpha \beta \gamma}$. Now with much the same logic as the proof of Claim 3.10, and modulo sub-sequencing: $v_{n}$, resp. $v_{n}^{\prime}$, resp. $v_{n}^{\prime \prime}$ converge to $v$, resp. $v^{\prime}$, resp. $v^{\prime \prime}$, so from $X$ separated, $a_{n}$, resp. $b_{n}$, converges to $a$, resp. $b$, and, whence, since $R$ is separated, $A_{n}$, resp. $B_{n}$ converges to $F(a)$, resp. $F(b)$, while $(a \times F(a)) \times(b \times F(b)) \in T_{\alpha \beta \gamma}$, which is absurd.

Now we can return to the proof of Fact 3.9. Plausibly $T$ isn't a groupoid, but it contains a subset which is as soon as the conclusions of Claims 3.10-3.12 hold, to wit if $j: T \rightarrow G$ is the projection, we have a sub-groupoid of $G \times R$ defined by,

$$
\begin{aligned}
T_{G}:=\left\{f \in T \mid T \cap t^{-1}(s(f)) \rightarrow t^{-1}(s(j f)),\right. \\
\text { and, } \left.T \cap s^{-1}(t(f)) \rightarrow s^{-1}(t(j f))\right\} .
\end{aligned}
$$

Indeed if $f \in T_{G}$ then whenever Claim 3.11 holds the only arrows in $T$ projecting to identities in $G$ are themselves identities in $G \times R$, so, by definition, (43), $T_{G}$ contains identities, while by the symmetry in (43) it also contains inverses. Finally if $g, f \in T_{G}$ lying over a composition in $V_{a} \times{ }_{X} V_{\beta} \times{ }_{X} V_{\gamma}$ are composable then under the conclusion of Claim 3.12 the result of the composition, although it may not belong to $T_{\alpha \gamma}$, does belong to a slightly larger open set on which the source is injective provided that Claim 3.10 holds too. On the other hand the unique - in the presence of Claim 3.10 - arrow in $T_{\alpha \gamma}$ which can be composed with $f$ is already in $T_{G}$ by construction.

Possibly $T_{G} \hookrightarrow T$ isn't open, but the interior of a groupoid is a groupoid, and the interior of $T_{G}$ contains the graph of the functor $F$, as will emerge in the proof of,

Claim 3.13 If $T_{0}$ is sufficiently small, then the interior of $T_{G}$ is a separated groupoid.

Proof of Claim 3.13 Again fix a compact set $K$ as in (38) in the proof of Claim 3.10, and suppose that for a sequence of open neighbourhoods $T_{0}^{n}$ collapsing to the graph of $K$, with associated $T^{n}$ as in (37) et seq. resulting in $T_{G}^{n}$ defined by (43), that for $I_{0}^{n} \hookrightarrow T_{0}^{n}$ the objects of the interior there is a sequence of arrows $g_{m}^{n}$ which converge to $g_{n} \in I_{0}^{n} \times I_{0}^{n}$ but not in the interior of $T_{G}^{n}$ as $m \rightarrow \infty$. Consequently, sub-sequencing in $n$, for each $n$ we can take $m(n) \gg 0$ such that the image of $g_{m(n)}^{n}$ converge to a 
point in $K \times K$, and whence, since $X$ is separated, their images $j\left(g_{m(n)}^{n}\right)$ converge to an arrow $g \in G$, so that, finally, $g_{m(n)}^{n}$ converge to $g \times F(g)$. Now provided we chose $m(n)$ sufficiently large, this is equally the limit of the $g_{n}$. It therefore remains to analyse the structure of $T_{G}$ around $g$. To this end we can choose a sequence of neighbourhoods $N_{\alpha}^{n}$, resp. $N_{\beta}^{n}$, of the source, resp. the sink, of $g$ which via the sink, resp. the source, are isomorphic to some neighbourhood $N_{\alpha \beta}^{n} \ni g$ in $V_{\alpha} \times{ }_{X} V_{\beta}$. Moreover, since the cover $V$ is locally finite, if an arrow $g_{\gamma \alpha} \in V_{\gamma} \times{ }_{X} V_{\alpha}$, resp. $g_{\beta \gamma} \in V_{\beta} \times{ }_{X} V_{\gamma}$, can be composed with $g$ then there is an inverse, $t^{-1}$, of the sink, resp. $s^{-1}$ of the source, over $N_{\alpha}^{n}$, resp. $N_{\beta}^{n}$. Similarly we can suppose that $\Omega_{\gamma \alpha}$, resp. $\Omega_{\alpha \beta}$, resp. $\Omega_{\beta \gamma}$, is an open neighbourhood of $F\left(g_{\gamma \alpha}\right)$, resp. $F(g)$, resp. $F\left(g_{\beta \gamma}\right)$ around which the source and sink of $R$ are homeomorphisms, and $t^{-1} N_{\alpha} \subseteq F^{-1}\left(\Omega_{\gamma \alpha}\right)$, resp. $s^{-1} N_{\alpha} \subseteq F^{-1}\left(\Omega_{\alpha \beta}\right)$, resp. $t^{-1} N_{\beta} \subseteq F^{-1}\left(\Omega_{\alpha \beta}\right)$, resp. $s^{-1} N_{\beta} \subseteq F^{-1}\left(\Omega_{\beta \gamma}\right)$. It therefore follows that, without loss of generality, $N_{\alpha}^{n} \times s\left(\Omega_{\alpha \beta}\right)$, resp. $N_{\beta}^{n} \times t\left(\Omega_{\alpha \beta}\right)$ are open neighbourhoods of the source, resp. sink, of $g$ in $I_{0}^{n}$ which via the source, resp. the sink, are isomorphic to $N_{\alpha \beta}^{n} \times \Omega_{\alpha \beta}$. In particular, if $g_{m}^{n}$ converge, $m \rightarrow \infty$, in $I_{0}^{n} \times I_{0}^{n}$ then they converge in $N_{\alpha \beta}^{n} \times \Omega_{\alpha \beta}$.

Now we can complete the proof in the obvious way. Specifically choose $T$ sufficiently small that (3.13) holds, so that in the notation of the proof of op. cit. we have a separated groupoid,

$$
\operatorname{int}\left(T_{G}\right) \rightrightarrows I_{0} \hookrightarrow T_{0}
$$

As such the classifier of (44) is a separated manifold, $M$, which by construction is an open sub-groupoid of $G \times R$ containing the graph of $F$, so we get maps,

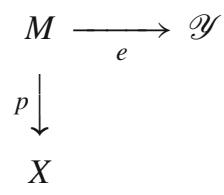

together with a section $\sigma$ of $p$ such that $e \sigma=f$ and $p \times e$ is étale. To conclude, therefore, we need only observe that by construction, the normal bundle to $\sigma(X)$ is isomorphic to $f^{*} T_{\mathscr{Y}}$.

The most useful applications of transversality are to multi-jets, which is a minor variation, i.e.

Definition 3.14 Let $\mathscr{X}$ be a differentiable champ and $m \in \mathbb{Z}_{>0}$, then we denote by $\mathscr{X}^{(m)}$ the complement in $\mathscr{X}^{m}$ of the image of all diagonals, be they big or small, which, as we've already seen, (15), is a bit bigger than one might think, i.e. for the classifier $[U / G]$ of a group action, $[U / G]^{(2)}$ is locally in $U^{2}$ the complement of all of $\left\{\left(u, u^{g}\right): g \in G\right\}$. In any case $j: \mathscr{X}^{(m)} \rightarrow \mathscr{X}^{m}$ is an open embedding, and for $\mathscr{Y}$ another differentiable champ we define,

$$
J_{r}^{m}(\mathscr{X}, \mathscr{Y}):=J_{r}(\mathscr{X}, \mathscr{Y})^{m} \times \mathscr{X}^{m} \mathscr{X}^{(m)}, \quad r \in \mathbb{Z} \geqslant 0 .
$$


Similarly if $E \rightarrow \mathscr{X}$ is a vector bundle, and $E_{i} \rightarrow \mathscr{X}^{(m)}$ is the pull-back along the inclusion $j$ followed by the projection to the $i$ th-factor,

$$
J_{r}^{m}(E):=J_{r}\left(\coprod_{1 \leqslant i \leqslant m} E_{i}\right) .
$$

\section{Transversality}

To apply this to transversality via finite dimensional linear algebra we need the well known,

Lemma 4.1 Let $U \subseteq X$ be a relatively compact open subset of a separated manifold $X$, and $E \rightarrow X$ a vector bundle on the same, then there is a finite dimensional vector space $V \subset \mathrm{H}^{0}(U, E)$ of sections which separate $(m, r)$ multi-jets over $U$, i.e.

$$
V \rightarrow \prod_{1 \leqslant i \leqslant m} E / \mathfrak{m}\left(x_{i}\right)^{(r+1)} \text {, is surjective } \forall \underline{x}=\left(x_{i}\right) \in U^{(m)},
$$

where $U^{(m)}$ is as per Definition 3.14.

Proof We can suppose that $U$ is covered by finitely many open sets $U_{\alpha} \Subset \mathbb{R}^{n}$. Furthermore if $E$ were functions on $\mathbb{R}^{n}$ then the proposition is true for a space of polynomials of sufficiently large degree since any coherent sheaf on a projective space is generated by sections after twisting by a sufficiently large power of the tautological bundle, and we can reduce the general case to this case since by way of appropriate bump functions the evaluation maps in (45) can be considered independently over $U_{\alpha}^{m_{\alpha}(\underline{x})}$, where $m_{\alpha}(\underline{x})$ is the number of $x_{i}$ contained in $U_{\alpha}$.

The generalisation of this to champ is, in a sense unsatisfactory, but at the same time it's best possible, to wit:

Lemma 4.2 Let $\mathscr{U} \subseteq \mathscr{X}$ be a relatively compact open subset of a differentiable separated champ $\mathscr{X}$, and $E \rightarrow X$ a vector bundle on the same, then there is a finite dimensional vector space $V \subset \mathrm{H}^{0}(\mathscr{U}, E)$ of sections which separate $(m, r)$ multi-jets over $\mathscr{U}$ quantum potest, i.e.

$$
V \rightarrow \prod_{1 \leqslant i \leqslant m}\left(E / \mathfrak{m}\left(x_{i}\right)^{(r+1)}\right)^{A_{x_{i}}}, \text { is surjective } \forall \underline{x}=\left(x_{i}\right) \in \mathscr{U}^{(m)}
$$

where $A_{x}=\operatorname{Aut}_{\mathscr{X}}(x)$ is the local monodromy of a point.

Proof As before we cover $\mathscr{U}$ by finitely many things that we understand, i.e. classifiers $\left[U_{\alpha} / G_{\alpha}\right]$ of finite group actions where $U_{\alpha} \Subset \mathbb{R}^{n}$ and $G_{\alpha} \subset \operatorname{GL}(n, \mathbb{R})$ acts linearly. In particular there is an embedding $\left[U_{\alpha} / G_{\alpha}\right] \hookrightarrow\left[\mathbb{P}_{\mathbb{R}}^{n} / G_{a}\right]$ in a projective algebraic champ, $\mathscr{P}$. Now ample bundles, $H$, on projective champ in characteristic zero need 
not separate jets, but they are co-homologically ample, i.e. for every coherent sheaf $\mathscr{F}$,

$$
\mathrm{H}^{q}\left(\mathscr{P}, \mathscr{F} \otimes H^{n}\right)=0, \quad \forall n \gg 0, \quad q>0
$$

which implies (46) for $\left[\mathbb{R}^{n} / G_{\alpha}\right]$, while partitions of unity exist, [8, 3.9], so we may conclude exactly as in Lemma 4.1.

The relevance of this is,

Fact 4.3 If $V$ is a finite dimensional vector space of sections of a vector bundle $E$ on a differentiable champ $\mathscr{X}$ which separates $(m, r)$ multi-jets then the evaluation map,

$$
e: V \times \mathscr{X}^{(m)} \rightarrow J_{r}^{m}(E): s \mapsto j_{r}(s)^{m}
$$

is smooth, 0.5.

Proof By construction, (29), $e$ of (47) is just a map of vector bundles, and the surjectivity condition (45) guarantees that the sheaf kernel of (47) is in fact an injection of vector bundles, which is what we need for a submersion.

Before proceeding to some transversality statements it remains to address the possibility that $\mathscr{X}$ is non-compact by way of the following set up, which may be ignored at a first reading.

Set Up 4.4 Let $\mathscr{U}_{n} \subseteq \mathscr{U}_{n+1} \subseteq \mathscr{X}$ be an exhaustion of a separable and separated differential champ $\mathscr{X}$ by an ascending chain of countably many relatively compact opens. As such if $E \rightarrow \mathscr{X}$ is a vector bundle then we may suppose,

(a) $\exists V_{n} \subset \mathrm{H}^{0}\left(\mathscr{U}_{n}, E\right)$, a finite dimensional vector space satisfying (46) over $\mathscr{U}_{n}$.

(b) The image under restriction of $V_{n+1}$ is exactly $V_{n}$.

Indeed if $\rho$ is identically 1 on $\mathscr{U}_{n}$ with compact support in a slightly bigger open $\mathscr{U}_{n}^{\prime}$ over which the $V_{n}$ still satisfies (46) then we need to find a finite dimensional space of section $W$ of some slightly bigger $\mathscr{U}_{n+1}^{\prime} \supset \mathscr{U}_{n+1}$ such that sections of the form,

$$
(1-\rho) w+\rho v, \quad w \in W, \quad v \in V_{n}
$$

satisfy (46) over $\mathscr{U}_{n+1}$. The condition is however open in $\left(\mathscr{U}_{n+1}^{\prime}\right)^{m}$, so if we couldn't do (b) it would be because there would be a point where (46) failed for every finite dimensional $W$ which by the finite dimensionality of the right hand side of (46) and the existence of partitions of unity is absurd. This said observe that the sheaf condition gives a natural embedding,

$$
{\underset{\lim }{n}}_{n} V_{n} \hookrightarrow \mathrm{H}^{0}(\mathscr{X}, E)
$$


and we're interested in properties $P_{n}(x, s)$ of $s \in K_{n} \subset V_{n}$ at $x \in \mathscr{U}_{n}$ where $K_{n+1} \rightarrow$ $K_{n}$ is a surjective chain of compacts such that,

(1) $P_{n}(x, s) \Rightarrow P_{l}\left(x, s \mid \mathscr{U}_{l}\right)$ whenever $x \in \mathscr{U}_{l}, l \leqslant n$.

(2) The set $Z_{n}:=\left\{s \in K_{n} \mid P_{n}(x, s)\right.$ fails $\left.\forall x \in \mathscr{U}_{n}\right\}$ has zero Lebesgue measure.

Now $K=\lim _{n} K_{n}$ is compact, so after appropriate scaling of Lebesgue measure on the kernel of $V_{n+1}^{\longleftarrow} \rightarrow V_{n}$ we can suppose $K$ is a probability space, while if $p_{n}: K \rightarrow Z_{n}$ is the projection then $p_{n}^{-1}\left(Z_{n}\right)$ has zero measure, and whence

$$
K \backslash \bigcup_{n} p_{n}^{-1}\left(Z_{n}\right)
$$

has measure 1.

In practice $E$ will be the pull-back of the tangent bundle from another differentiable champ, and the definition of $K_{n}$ will be the closure of,

$$
K_{n}^{\prime}:=\left\{s \in V_{n}: s \text { factors through } M \text { in (34) }\right\} .
$$

Plainly we're free to suppose that the fibres of $M$ (via the first projection in (34)) are compact balls, so the $K_{n}$ are certainly compact, albeit one might express a doubt as to whether $K_{n+1} \rightarrow K_{n}$ is surjective. However, from (48) there are extensions of any section in $K_{n}$ to $\mathscr{U}_{n+1}$ which are as small as we need.

Now the easiest way to distinguish the wood from the trees in Proposition 1.1, is to first restrict attention to finite dimensional deformation spaces or limits thereof, via Lemma 4.1 and the Set Up 4.4, to wit:

Proposition 4.5 Let $f: X \rightarrow \mathscr{Y}$ be a map from a separated manifold to a differentiable champ admitting an exponential map, Definition 3.8, from a neighbourhood of the zero section of the pull-back of the tangent space, and $m \in \mathbb{Z}_{>0}, r \in \mathbb{Z} \geqslant 0$ then there is a compact probability space, $K$, of deformations of $f$ (in fact just a ball in a space of sections separating $m, r$ multi-jets of $f^{*} T_{\mathscr{Y}}$ if $X$ is compact, or even just finite type, and as per the Set Up 4.4 otherwise) such that if $\mathscr{Z} \rightarrow J_{r}^{m}(X, \mathscr{Y})$ is a representable separated net map from a (smooth) differentiable champ of co-dimension $q$ to the space of multi-jets, Sect. 3, enjoying a property $(\mathrm{P})$ stable under base change, then for almost all $g \in K$, the left hand vertical of the fibre square,

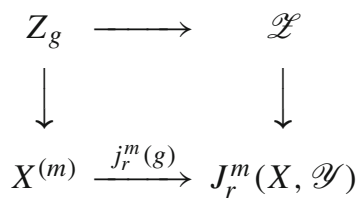

is empty or a net map enjoying property (P) from a separated manifold, whence, in particular, empty if $q$ exceeds the dimension of $X$. 
Proof We do the case $X$ compact since the Set Up 4.4 has been constructed so that the general case just goes through mutatis mutandis. In any case by (32) and (33) we can replace $\mathscr{Z}$ by its fibre under $j_{r}^{m}(\exp )$, i.e. for $M$ the domain of the exponential of Definition 3.8, we have a diagram, of fibre squares,

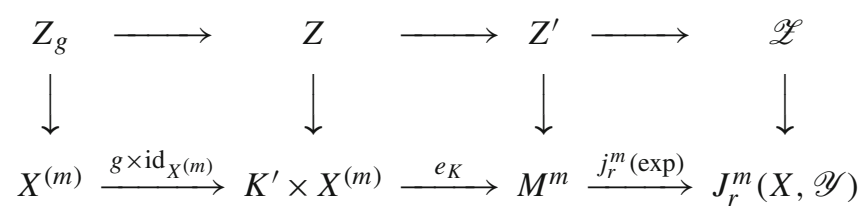

where, $K^{\prime} \subset K$ is as per (49) and whence evaluation factors through the domain of the exponential. As such if the property $(\mathrm{P})$ is stable under base change, all the verticals in (51) have property $(\mathrm{P})$. Furthermore the lower rightmost horizontals are, from the right, étale, resp. smooth, whence idem for the top two so $Z$ is certainly a differentiable champ. By hypothesis, however, the rightmost vertical is also representable so $Z$ is a manifold. Our definitions, (7), however allow this to be understood in the broadest sense, so, a priori, manifold here only means B-manifold. Nevertheless the rightmost vertical in (51) is net, so there is a presentation in $\underline{B}$,

$$
R \rightrightarrows U=\coprod_{\alpha \in A} U_{\alpha}
$$

such that for every $\alpha$, the aforesaid arrow maps $U_{\alpha}$ diffeomorphically to its image in $K^{\prime} \times X^{(m)}$. Consequently, by the universal property of $U \rightarrow(U / R)_{\text {Top }}$, its restriction to $U_{\alpha}$ is a homeomorphism onto its image. Thus $Z$ is a manifold in the text book sense which is separated as soon as the right vertical of (51) is, since this ensures that its fibres over the separated space $K^{\prime} \times X^{(m)}$ are, themselves, separated. Better the projection,

$$
Z \rightarrow K^{\prime}
$$

is a map of infinitely differentiable separated manifolds, so by Sard's theorem almost every fibre is smooth, but the fibre over $g \in K^{\prime}$ is exactly the pre-image of $\mathscr{Z}$ under (50), which preserves the codimension by a special case of the 9-lemma, cf. (53).

Before adding the infinite dimensional gloss via Smale's variant of Sard's theorem, let us observe,

Remark 4.6 The intervention of Sard's theorem is the only point where we're using that the right vertical in (50) is separated. Equally, although Sard's theorem is false for non-separated manifolds, even those in the text book sense, it requires something ridiculous for it to fail. Specifically, one could start from a holomorphic degeneration $X \rightarrow \Delta$ of a Riemann surface $\Sigma$ parametrised by the disc with singular fibre a nodal curve, then slice along an interval $I \hookrightarrow \Delta$ through the origin 0 to get a 3-manifold $M \rightarrow I$ whose fibre over $I \backslash\{0\}$ is a product with $\Sigma$. As such, for any $j$ in another interval $J$ one can glue $M$ to $\Sigma \times J$ by identifying $I \backslash\{0\}$ and $J \backslash\{j\}$ to get a nonseparated manifold with a singular fibre over $j$, then just repeat this for $j$ in a set of 
positive measure to get a counter example to Sard's theorem. On the other hand, since Sard's theorem is locally true, this is also a typical counterexample, so the degree of pathology required to make counterexamples is well beyond anything one might meet in practice as explained in Definition 2.6, i.e. representable maps in separated manifolds are a fortiori separated.

This said, let's address the intervention of Smale's variant of Sard, by way of,

Scholion 4.7 In the first place say $X$ compact, to facilitate the initial discussion, then $K$ in Proposition 4.5 is a residual (countable intersection of open dense sets) subset of a ball in any finite dimensional subspace, $V$, of $\mathrm{H}^{0}\left(X, f^{*} T_{\mathscr{Y}}\right)$ separating $m, r$ multi-jets since the set of critical points is both of measure zero and a countable union of closed sets. The residual statement is Smale's formulation, [16, 1.3], of Sard's theorem and it allows the proof to work for any closed subspace $V$ of $\mathrm{H}^{0}\left(X, f^{*} T_{\mathscr{Y}}\right)$, in the usual (i.e. bounded $C^{p}$-norm, $p$ any, on compact sets) Fréchet space structure of infinitely differentiable sections of bundles, separating $m, r$ multi-jets. Indeed the only point of caution is that things here are Fréchet rather than Banach, and while there are very general theorems extending Smale from Banach to Fréchet, the situation here is so simple that it's just a diagram chase to check that Smale's argument, [16, 1.5 et seq.], works. Specifically we put ourselves at a point $z=(v, x) \in Z$, in the top, 2nd from the left entry in (51), but with $K^{\prime}$ now an open neighbourhood in the possibly infinite dimension space $V$, and, of course, the evaluation $e$ (middle horizontal) is still smooth by our multi-jet hypothesis. Now, the discussion is local about $z$ and,

(a) We apply the finite dimensional implicit function theorem to the second vertical from the right in (51) so as to suppose that it's an inclusion of open neighbourhoods of zero in finite dimensional vector spaces. In particular we identify the inclusion $\{x\} \hookrightarrow X^{(m)}$ with that of the origin in a finite dimensional vector space.

(b) Since it's a fibre of a finite dimensional map, [9], it's also true that $Z$ around $z$ is isomorphic to an open neighbourhood of a closed linear subspace of $V \times X^{(m)}$ of finite co-dimension, or, better for our purposes, a quotient by a finite dimensional subspace, cf. (52).

(c) The key point, (54), involves some choices, so it's useful to make the isomorphism in (b) as clean as possible. The relevant diagram of tangent spaces at $z$ is given by (53) wherein $T, Q, S, C$ are defined by the diagram. In particular a convenient choice for the isomorphism (b) is to choose a section $v$ of the quotient in the middle row of (53) so as to get a composition,

$$
Z \hookrightarrow V \times X^{(m)} \stackrel{p}{\longrightarrow} V \times X^{(m)} / v\left(f^{*} N_{\mathscr{Z}} / \mathscr{Y}\right)
$$

in which the quotient $p$ is understood according to the linearisation (a) of $X^{(m)}$.

(d) The section $v$ can be chosen to be compatible with the splitting in the middle row of (53), i.e. a section of the rightmost entry of the bottom row equally gives a splitting of the rightmost column, so a further choice of a section splitting the top row gives identifications,

$$
V \times X^{(m)} \stackrel{\sim}{\longrightarrow}(S \coprod C) \times(T \coprod Q), \quad \text { and }, \quad Z=\{(s, c(s, t)) \times(t, q(s, t))\}
$$


for some infinitely differentiable functions $c$ and $q$.

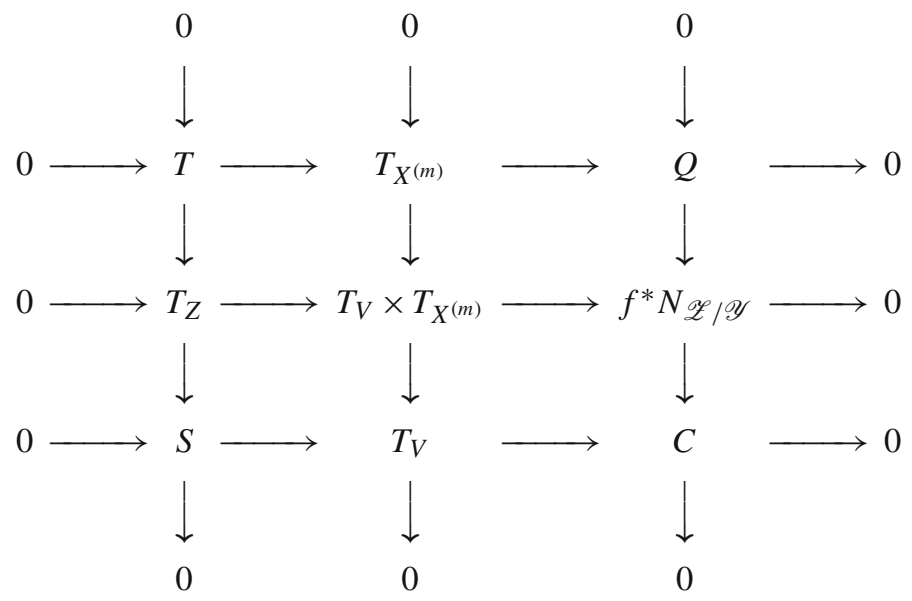

(e) Finally therefore we get a commutative diagram,

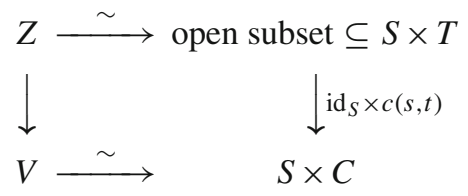

with $C, T$ finite dimensional, which is the key point, [16, proof of 1.3], in Smale's reduction of his infinite dimensional Sard theorem to the finite dimensional one.

As such, for $X$ compact Proposition 4.5 is true as stated for

Extra Fact 4.8 $K$ a residual subset of an open neighbourhood of the origin in a closed subspace of $\mathrm{H}^{0}\left(X, f^{*} T_{\mathscr{Y}}\right)$ separating $m, r$ multi-jets.

In the case that $X$ isn't compact then the only real change is that we can't use the usual Fréchet topology on $\mathrm{H}^{0}\left(X, f^{*} T_{\mathscr{Y}}\right)$. More specifically if say the vector spaces $V_{n}$ in Set Up 4.4 were now the image of global sections in $\mathrm{H}^{0}\left(\mathscr{U}_{n}, f^{*} T_{\mathscr{Y}}\right)$ and we define $K_{n}^{\prime}$ as in (49), then the pre-image of each $K_{n}^{\prime}$ in $\mathrm{H}^{0}\left(X, f^{*} T_{\mathscr{Y}}\right)$ is as near to an open $K_{n}^{\prime \prime}$ (i.e. same condition as (49) but on the closure of $\mathscr{U}_{n}$ ) as makes no difference. Ultimately, however, we'll need global sections which lie in every $K_{n}^{\prime \prime}$, and their intersection may well fail to be open. Fortunately, the problem is no worse than this, while the only functional analysis we used in (a)-(e) was the implicit function theorem for maps to finite dimensional spaces, which is true, [9], even for locally convex spaces so all we need to do is to take,

Set Up 4.9 The coarsest locally convex topology on $\mathrm{H}^{0}\left(X, f^{*} T_{\mathscr{Y}}\right)$ containing the usual Fréchet topology in which the intersection of the pre-images of all of the above $K_{n}^{\prime \prime}$ is open.

Now (a)-(e) apply as stated but with the topology of Set Up 4.9, so that for $X$ any separated manifold, 
Extra Fact 4.10 For any closed subspace, $V$, of $\mathrm{H}^{0}\left(X, f^{*} T_{\mathscr{Y}}\right)$ in the topology of the Set Up 4.9 which separates $m, r$ multi-jets Proposition 4.5 is true for a space of deformations parametrised by a residual subset of an open neighbourhood of the origin in $V$.

Equally it can be possible to use Lemma 4.2, in the finite dimensional context, or more generally, Extra Fact 4.10, and get results for maps from orbifolds, or, more generally, separated champs. Invariably, however, such results are more ad hoc, e.g.,

Remark 4.11 If the domain of $f$ in Proposition 4.5 is a differentiable separated champ rather than a separated manifold then transversality valeat quantum potest valere, i.e. as much as the difference between (45) and (46) allows. Specifically if the action of the local monodromy on the right hand side of (46) is non-trivial then Fact 4.3 fails, so $e_{K}$ in (51) won't be smooth, and the entry $Z$ in op. cit. will fail to be a differentiable champ. The failure here is, however, far from catastrophic, and for best results one should eschew the use of Sard's theorem, cf. [2]. For example to do the case of Morse functions without effort observe that if $\mu: \mathscr{X} \rightarrow X$ is the moduli, Fact/Definition 2.7, of a differentiable separated champ then for $n \in \mathbb{Z} \geqslant 0$ the set

$$
X_{n}=\left\{x \in X \mid \operatorname{card}\left(A_{x}\right) \geqslant n\right\}, \quad A_{x}:=\operatorname{Aut} \mathscr{X}(x) .
$$

is closed, and the pre-image, $\mathscr{Z}_{n}$, of $Z_{n}:=X_{n} \backslash X_{n+1}$ is a differentiable champ while $Z_{n}$ itself is a manifold. In particular therefore, for $z \in Z_{n}$ there is an exact sequence

$$
0 \rightarrow T_{\mathscr{Z}_{n}}(z) \rightarrow T_{\mathscr{X}}(z) \rightarrow N_{\mathscr{Z}_{n}} / \mathscr{X}(z) \rightarrow 0
$$

Now for convenience we again suppose $\mathscr{X}$ compact (and otherwise as per Set Up 4.4) so we can take a finite dimensional space $V$ of functions separating 2-jets in quantum potest, i.e. (46) is valid. As such the conditions,

$$
F_{n}:=\left\{(f, z)|d f|_{T_{\mathscr{Z}_{n}}(z)}=0\right\} \hookrightarrow V \times \mathscr{Z}_{n}
$$

define a sub-bundle of the restriction of $V$ to $\mathscr{Z}_{n}$ of co-dimension over a connected component of $\mathscr{Z}_{n}$ the dimension of the same. In consequence the dimension of $F_{n}$ is that of $V$ and it may well surject onto the latter. On the other hand if $(f, z) \in F_{n}$ then since the representation of the local monodromy on the normal bundle in (55) has no fixed vectors, $d f$ vanishes on all of $T_{\mathscr{X}}(z)$ so there is a well defined Hessian,

$$
H_{f}(z): \mathfrak{m}(z) / \mathfrak{m}(z)^{2} \times \mathfrak{m}(z) / \mathfrak{m}(z)^{2} \rightarrow \mathbb{R}
$$

and we may consider

$$
E_{n}:=\left\{(f, z) \mid H_{f}(z)=0\right\} \hookrightarrow F_{n}
$$

which is itself a sub-bundle since $V$ satisfies (46) and the local monodromy representations are linearisable. Finally observe that at every point $z \in \mathscr{Z}_{n}$, there is a strict inclusion

$$
E_{n}(z) \subset F_{n}(z)
$$


of the fibres of (56) over $z$, since, inter alia, there are, locally about $z$, invariant squared distance functions associated to $A_{z}$ invariant metrics. Consequently for every $n \geqslant 0$ the dimension of the bundles $E_{n}$ of (56) is at most 1 less than the dimension of $V$, i.e. the set of $f \in V$ which have degenerate critical values on $\mathscr{Z}_{n}$ for some $n$ is not only of measure 0 but the image of finitely (or countable if we hadn't supposed compactness) many differentiable maps from manifolds of strictly smaller dimension.

\section{A Guide to the examples}

We begin by expanding on the introduction, to wit,

Fact A.1 Let $L \stackrel{\pi}{\rightarrow}\left[\Delta / \mu_{n}\right]$ be a holomorphic line bundle over a disc with a nonorbifold point of order $n$ defined by a character $\chi$, i.e. $\mu_{n}$ acts on $\Delta$ by a rotation of order $n$, and, on (canonically) identifying $\chi$ with $a \in \mathbb{Z} / n, L$ is the classifier of the diagonal action,

$$
\mathbb{G}_{a} \times \Delta:(e, z) \mapsto\left(\theta^{a} e, \theta z\right) \quad \theta \in \mu_{n},
$$

then for any $m \in \mathbb{Z}_{>0}$, and any infinitely differentiable section $s:\left[\Delta / \mu_{n}\right] \rightarrow L^{\oplus m}$ the pre-image of the ideal of (differentiable) functions vanishing on the zero section is contained in the ideal,

$$
\left(z^{i} \bar{z}^{j} \mid j=i+a(n)\right), \quad i, j \in \mathbb{Z} \geqslant 0
$$

Proof The ideal (57) contains all asymptotically flat functions, i.e. those with trivial Taylor expansion. As such it is sufficient to prove the statement after completing in the origin, i.e. modulo flat functions. Consequently we can identify $s$ with an $m$-tuple of Taylor series,

$$
\left(f_{1}, \ldots, f_{m}\right) \in \mathbb{C}[[z, \bar{z}]]^{\oplus m}
$$

which is invariant for the action of $\mu_{n}$, i.e. for all $1 \leqslant k \leqslant m$,

$$
\left(f_{k} \cdot e\right)^{\theta}=f_{k} \cdot e, \quad \text { which is iff } \theta^{a} f_{k}\left(\theta z, \theta^{-1} \bar{z}\right)=f_{k}(z, \bar{z}) .
$$

Thus each $f_{k}, 1 \leqslant k \leqslant m$ actually belongs to the ring,

$$
\mathbb{C}\left[\left[z^{i} \bar{z}^{j} \mid j=i+a(n)\right]\right] \subset \mathbb{C}[[z, \bar{z}]]
$$

while the ideal in question is that generated by the $f_{k}$ so (57) follows a fortiori from (58).

Next, in order to distinguish the general from the particular it's best to expand the example of the B-manifold $\mathbb{R} / \mathbb{Q}$ of Fact/Definition 2.4.(d) to any discrete group $\Gamma$ acting faithfully by translations on $\mathbb{R}$, and to this end we'll need, 
Lemma A.2 Suppose $\Gamma$ acts faithfully on $\mathbb{R}$ by translations, and for $\epsilon>0$ let $\Gamma_{\epsilon}$ be the subgroup generated by elements of modulus at most $\epsilon$ then,

$$
\Gamma_{\epsilon}= \begin{cases}0 & \text { if } \Gamma \stackrel{\sim}{\rightarrow} \mathbb{Z}, \text { and } \epsilon \text { is sufficiently small, } \\ \Gamma & \text { otherwise. }\end{cases}
$$

Proof Suppose there exists $\beta \in \Gamma \backslash \Gamma_{\epsilon}$, then,

$$
\Gamma^{\prime}:=\{\gamma \in \Gamma \mid n \gamma \in \mathbb{Z} \beta, 0 \neq n \in \mathbb{Z}\}
$$

must be generated by some $\beta / n$, so, without loss of generality $\Gamma^{\prime}=\mathbb{Z} \beta$. On the other hand $\Gamma / \Gamma^{\prime}$ is torsion free, so, either its zero, and we're done, or there is a rank 2 submodule of $\Gamma$ containing $\Gamma^{\prime}$, so it'll suffice to prove the lemma for $\Gamma=\mathbb{Z}^{2}$. Now by Dirichlet's application of the pigeon hole principle, $\Gamma_{\epsilon}$ has rank 2 , and is dense in $\mathbb{R}$, so there is a $\gamma \in \Gamma_{\epsilon}$ such that,

$$
|\beta-\gamma|<\epsilon
$$

so, a fortiori, $\beta-\gamma \in \Gamma_{\epsilon}$.

Now the failure, Fact/Definition 2.4.(d), of $\mathbb{R} / \mathbb{Q}$ to be a manifold in the text book sense, equally extends to $\mathbb{R} / \Gamma$ for any non-trivial group of translations other than $\mathbb{Z}$ due to the crude intervention of point set topology in Fact/Definition 2.4.(a), which in the first instance manifests itself by,

Lemma A.3 Suppose a discrete group $\Gamma$ acts freely on a topological space $U$ then $h: U \rightarrow(U / \Gamma)_{\text {Top }}$ is a local homeomorphism iff it's a $\Gamma$-torsor, i.e. every point in the quotient has a neighbourhood, $V$, whose fibre is topologically $V \times \Gamma$.

Proof Sufficiency is clear. Conversely choose a point $u \in U$ above a given point in the quotient, then there is an open neighbourhood $V \ni u$ such that $\left.h\right|_{V}$ is a homeomorphism. In particular, $\left.h\right|_{V}$ is a set theoretic injection, so, for $\gamma \in \Gamma$

$$
V \cap V^{\gamma}= \begin{cases}V, & \gamma=\mathrm{id}_{\Gamma} \\ \varnothing, & \text { otherwise }\end{cases}
$$

Thus the open subset $\bigcup_{\gamma \in \Gamma} V^{\gamma}$ is both homeomorphic to $h(V) \times \Gamma$ and the fibre over $h(V)$.

while in the case in point this is hopelessly false, to wit:

Fact A.4 For a discrete group $\Gamma$ acting on $\mathbb{R}$ by translations the fundamental group of the topological quotient $(\mathbb{R} / \Gamma)_{\text {Top }}$ is, in the notation of $(59), \Gamma / \Gamma_{\epsilon}$, i.e. if $\Gamma \neq \mathbb{Z}$, any torsor under a discrete group $G$ is trivial; whereas isomorphism classes of $G$ torsors on the $\underline{B}$-manifold $\mathbb{R} / \Gamma$ (or even the $\underline{T o p}_{\text {Ét }}$ sheaf $\mathbb{R} / \Gamma$ ) are classified by the cohomology set,

$$
\mathrm{H}^{1}(\Gamma, G):=\operatorname{Hom}_{\mathrm{Grp}}(\Gamma, G) /\left\{\phi \sim \psi \mid \exists g \in G, \psi=g \phi g^{-1}\right\}
$$


and, indeed, $\mathbb{R} \rightarrow \mathbb{R} / \Gamma$ is the universal cover, i.e. if $E \rightarrow \mathbb{R} / \Gamma$ is any connected $G$-torsor, then $G$ is a quotient of $\Gamma$ by some sub-group $\Gamma^{\prime}$, and $E$ is the $\underline{B}$ manifold $\mathbb{R} / \Gamma^{\prime}$ with $\Gamma^{\prime}$ acting by translations.

Proof Let $E \rightarrow(\mathbb{R} / \Gamma)_{\text {Top }}$ be a right $G$-torsor, and consider the diagram of fibred squares,

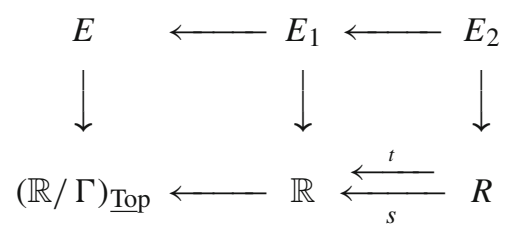

where $R$ is as in (9), i.e. the subset $\left\{\left(x, x^{\gamma}\right) \mid \gamma \in \Gamma\right\}$ of $\mathbb{R}^{2}$ in the induced topology with $s, t$ the projections. Now, $E_{1}$ is the trivial $G$-torsor, thus we may choose a section $\sigma$, which, in turn affords sections $s^{*} \sigma$ and $t^{*} \sigma$ of $E_{2}$ over $R$. In particular for each $f \in R$ there is a unique $T_{f} \in G$ such that,

$$
s^{*} \sigma(f)=t^{*} \sigma(f)^{T_{f}}
$$

and since $E_{2} \rightarrow R$ is a $G$-torsor, the assignment $T: f \mapsto T_{f}$ is a continuous map of $R$ to $G$. Consequently, for $\gamma \in \Gamma$, the restriction,

$$
T_{\gamma}:=A_{\gamma}^{*} T, \quad A_{\gamma}: \mathbb{R} \hookrightarrow R: x \mapsto(x, x+\gamma)
$$

is constant, while $A_{\beta} A_{\gamma}=A_{\beta+\gamma}$, so from the uniqueness in (62),

$$
T_{\beta} T_{\gamma}=T_{\beta+\gamma}
$$

and $T_{0}=\mathrm{id}_{G}$. However, $T$ is continuous, so the set where it takes the value $\mathrm{id}_{G}$ must contain a ball, so, a fortiori, there is $\epsilon>0$ such that,

$$
T_{\gamma}=\mathrm{id}_{G}, \quad|\gamma|<\epsilon
$$

and, by Lemma A.2, any such set generates $\Gamma$ if $\Gamma \neq \mathbb{Z}$, so, in fact $T=\operatorname{id}_{G}$ whenever $\Gamma \neq \mathbb{Z}$.

This proves that the equivalence relation $E_{2} \rightrightarrows E_{1}$ afforded by the fibres in (61) is (isomorphic) to the relation,

$$
R \times G \rightrightarrows \mathbb{R} \times G: x \times g \sim(x+\gamma) \times g, \quad \gamma \in \Gamma
$$

so, indeed, $E$ is trivial. At the same time, one plainly sees that the cause of this conclusion, (64), was the choice of topology on $R$, which is, of course, forced as soon as one (needlessly) insists that $\mathbb{R} / \Gamma$ should be understood as a topological space. If, however, we understand $\mathbb{R} / \Gamma$ as a $B$-manifold then for the same $E_{1}$ in (57), the 
definition of a B-manifold, Revision 2.2, implies that although $E_{2}$ in (57) remains the same set, $R$, its topology is that of the co-product,

$$
\coprod_{\gamma} \mathbb{R} \times \gamma
$$

As such, (64) fails, but all of (62)-(63) are valid, and whence $E_{2} \rightrightarrows E_{1}$ is the relation,

$$
R \times G \rightrightarrows \mathbb{R} \times G: x \times g \sim(x+\gamma) \times T_{\gamma} \cdot g, \quad \gamma \in \Gamma
$$

afforded by the diagonal action of the homomorphism defined by $T$ of (63). It therefore remains to observe the salient features of the definition of a B-manifold, to wit:

(a) The quotient of $\mathbb{R} \times G$ by (65) is the $\underline{B}$-manifold $\mathbb{R} \times G / \Gamma$ for the diagonal action of $\Gamma$.

(b) $\mathbb{R} \times G / \Gamma \rightarrow \mathbb{R} / \Gamma$ is certainly a $G$-torsor because its fibre over $\mathbb{R} \rightarrow \mathbb{R} / \Gamma$ is $\mathbb{R} \times G$, cf. Definition 2.6.

(c) There remains the question of when torsors defined by $T^{\prime}, T^{\prime \prime}$ in (65) are isomorphic, which by construction in (61) amounts to a $G$-map between two different relations, i.e. a map $\phi: \mathbb{R} \rightarrow G$ such that,

$$
T^{\prime} s^{*} \phi=t^{*} \phi T^{\prime \prime}
$$

and since any such $\phi$ must be constant, this is exactly (60).

(d) Finally let $\Gamma^{\prime}$ be the kernel of $T$ and $G^{\prime \prime}$ the image of $T$ in $G$, then (65) defines a relation on any left coset $\mathbb{R} \times g G^{\prime \prime}$, each of which is isomorphic to the same relation on $\mathbb{R} \times G^{\prime \prime}$, so, as a B-manifold, $E$ is a direct sum of $G^{\prime \prime}$-torsors indexed by $G / G^{\prime \prime}$. Thus connectedness implies $G=G^{\prime \prime}$, while conversely, slicing

$$
\Gamma \times\left(\mathbb{R} \times G^{\prime \prime}\right) \rightrightarrows \mathbb{R} \times G^{\prime \prime}
$$

along $\mathbb{R} \times \operatorname{id}_{G^{\prime \prime}}$ shows that it's equivalent to $\mathbb{R} / \Gamma^{\prime}$, which is certainly connected because $\mathbb{R}$ is.

At this juncture, it should be clear that the properties of the $\underline{B}$-manifold $\mathbb{R} / \Gamma$ are, as they should be, exactly the $\Gamma$-equivariant properties of $\mathbb{R}$, which, in turn, have absolutely nothing to do with $(\mathbb{R} / \Gamma)$ Top unless $\Gamma \stackrel{\sim}{\rightarrow} \mathbb{Z}$. Thus an instructive complement to Fact A.4 is provided by,

Definition A.5 Let $X$ be a B-manifold, or, more generally, a differentiable champ, of dimension $n$ then the De Rham cohomology, $\mathrm{H}_{\mathrm{DR}}^{\bullet}(X)$, of $X$ is the hyper-cohomology of the complex,

$$
\mathscr{A}_{X}^{0} \stackrel{d}{\longrightarrow} \mathscr{A}_{X}^{1} \stackrel{d}{\longrightarrow} \cdots \stackrel{d}{\longrightarrow} \mathscr{A}_{X}^{n-1} \stackrel{d}{\longrightarrow} \mathscr{A}_{X}^{n}
$$

where $\mathscr{A}^{p}$ is the sheaf of differential $p$-forms, which is actually a sheaf on $\underline{\mathrm{B}}_{\mathrm{E}}$, Definition 2.1, so a fortiori on $X$. In particular, (66) is quasi-isomorphic to the locally 
constant sheaf $\underline{\mathbb{R}}$, and whence there is a spectral sequence,

$$
\mathrm{E}_{1}^{p, q}=\mathrm{H}^{q}\left(X, \mathscr{A}^{p}\right) \Rightarrow \mathrm{H}_{\mathrm{DR}}^{p+q}(X) \stackrel{\sim}{\longrightarrow} \mathrm{H}^{p+q}(X, \underline{\mathbb{R}}) .
$$

Now, since there are several things to observe, let's gather them together in,

Scholion A.6 Evidently, this is not the usual definition of De Rham cohomology on manifolds in the text book sense, to wit the cohomology of,

$$
\mathrm{H}^{0}\left(X, \mathscr{A}_{X}^{0}\right) \stackrel{d}{\longrightarrow} \mathrm{H}^{0}\left(X, \mathscr{A}_{X}^{1}\right) \stackrel{d}{\longrightarrow} \cdots \stackrel{d}{\longrightarrow} \mathrm{H}^{0}\left(X, \mathscr{A}_{X}^{n-1}\right) \stackrel{d}{\longrightarrow} \mathrm{H}^{0}\left(X, \mathscr{A}_{X}^{n}\right)
$$

but this is a mistake already on a non-separated manifold in the text book sense, because it uses that (67) doesn't just degenerate at $E_{2}$ on separated manifolds, but even satisfies,

$$
\mathrm{E}_{1}^{p, q}=0 \text { if } q>0
$$

which is a consequence of the fact that separated manifolds admit partitions of unity, and whence $\mathscr{A}^{0}$-modules are acyclic. The $\underline{B}$-manifold, or more generally differentiable champ, situation is, however, more delicate. Specifically if a discrete group $\Gamma$ acts on a separated manifold, $U$, then a sheaf $\mathscr{F}$ on $[U / \Gamma]$ is a sheaf on $U$ with $\Gamma$-action, and nowhere does the Höschild-Serre spectral sequence need that $U \rightarrow(U / \Gamma)_{\text {Top }}$ is a local homeomorphism, so there is a spectral sequence,

$$
\mathrm{F}_{2}^{p, q}=\mathrm{H}^{p}\left(\Gamma, \mathrm{H}^{q}(U, \mathscr{F})\right) \Rightarrow \mathrm{H}^{p+q}([U / \Gamma], \mathscr{F}) .
$$

Thus, if we further suppose $U$ not just separated but also contractible, (67), simplifies to,

$$
\mathrm{E}_{1}^{p, q}=\mathrm{H}^{q}\left(\Gamma, \mathrm{H}^{0}\left(U, \mathscr{A}_{U}^{p}\right)\right) \Rightarrow \mathrm{H}_{\mathrm{DR}}^{p+q}([U / \Gamma])=\mathrm{H}^{p+q}(\Gamma, \mathbb{R}) .
$$

On the other hand if we put ourselves in the situation of Fact A.4, i.e. $U=\mathbb{R}$ and $\Gamma$ a group of translations, then (67) degenerates at $E_{2}$ for dimension reasons, so (69) is just the long exact sequence of $\Gamma$ co-homology associated to the short exact sequence of $\Gamma$-modules,

$$
0 \rightarrow \mathbb{R} \rightarrow \mathrm{H}^{0}\left(\mathbb{R}, \mathscr{A}_{\mathbb{R}}^{0}\right) \stackrel{d}{\longrightarrow} \mathrm{H}^{0}\left(\mathbb{R}, \mathscr{A}_{\mathbb{R}}^{1}\right) \rightarrow 0 .
$$

Now if $\Gamma \neq \mathbb{Z}$, the only invariant functions are the constants, and the only invariant differential is the derivative of the identity, so the long exact sequence associated to (70) starts,

$$
0 \rightarrow \mathbb{R} \stackrel{=}{\longrightarrow} \mathbb{R} \stackrel{d=0}{\longrightarrow} \mathbb{R} \stackrel{\delta}{\longrightarrow} \operatorname{Hom}(\Gamma, \mathbb{R}) \rightarrow \mathrm{E}_{\infty}^{0,1}=\mathrm{E}_{2}^{0,1} \rightarrow 0 .
$$

We may therefore draw the following negative conclusions,

(a) The complex (68) does not, in general, compute $\mathrm{H}_{\mathrm{DR}}^{\bullet}(\mathbb{R} / \Gamma)$.

(b) Indeed $\mathrm{E}_{\infty}^{0,1} \neq 0$ in the spectral sequence (69) as soon as $\Gamma$ has rational rank at least 2 , i.e. $\mathrm{H}_{\mathrm{DR}}^{1}(\mathbb{R} / \Gamma)$ can be arbitrarily bigger than the space of closed differential forms. 
(c) Consequently, $\mathbb{R} / \Gamma$, can have non-trivial $\mathbb{G}_{a}$-torsors, and, $\mathscr{A}^{0}$-modules needn't be acyclic, e.g. whenever $\mathrm{E}_{2}^{0,1}=\mathrm{E}_{\infty}^{0,1} \neq 0$.

On the positive side, albeit somewhat specific to the translation action, since in general $E_{2}^{1,0}=0$ in (69), one recovers the B-manifold from the aforesaid spectral sequence since the image of the differential of the identity under the connecting homomorphism $\delta$ in (71) is the inclusion of $\Gamma$ by its action on 0 .

Turning to how the main theorem, Proposition 1.1, applies to the examples of nonseparated manifolds and proper orbifolds encountered in Example/Definition/Warning 2.8 let us first recall,

Revision A.7 If $Y$ is a manifold in the text book sense, then the diagonal $\Delta: Y \rightarrow$ $Y \times Y$ is an embedded sub-manifold, i.e. $\Delta$ is a set embedding which is homeomorphic to its image, albeit $Y$ is separated iff $\Delta$ is also closed.

Proof The above definition of embedding is étale local, Definition 2.6, while from the definition of manifold in the text book sense, Fact/Definition 2.4.(a), $M$ admits a presentation,

$$
R \rightrightarrows U=\coprod_{\alpha \in A} U_{\alpha}
$$

where every $U_{\alpha} \rightarrow(U / R)_{\underline{T o p}}=Y$ is a homeomorphism onto its image. Now, plainly, the diagonal is always a set embedding, so, what has to be checked is that for all $\alpha, \beta \in A$, the topology on $U_{\alpha} \cap U_{\beta}$ is that induced by its inclusion into the product topology on $U_{\alpha} \times U_{\beta}$, which it is.

In particular, therefore, as a complement to the first example, (14), in Example/Definition/Warning 2.8,

Example A.8 Let $Y$ be a $q$-dimensional manifold in the text book sense, with $f: X \rightarrow$ $Y$ a map from a separated manifold then for almost any deformation $g$, i.e. belonging to the residual subset of Proposition 1.1, the fibre,

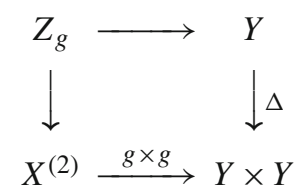

is empty or an embedded sub-manifold in the sense of Revision A.7 of co-dimension $q$, and, should $Y$ be separated then it's also closed.

Proof The diagonal map of a manifold in the text book sense, even a non-separated one, is separated, so we apply Proposition 1.1, with $r=0, m=2$, and (P) the étale local property: embedding in the sense of Revision A.7.

Now, the same example again, but this time as a complement to (15), 
Example A.9 Let $\mathscr{Y}$ be a $q$-dimensional differentiable champ with separated diagonal, e.g. the classifier $[Y / \Gamma]$ of a discrete group action on a possibly non-separated manifold in the text book sense, with $f: X \rightarrow Y$ a map from a separated manifold, then for almost all deformations $g$ of $f$, the fibre,

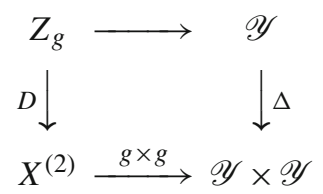

is either empty or a net map from a separated manifold of co-dimension $q$. Moreover if, the diagonal of $\mathscr{Y}$ is proper, i.e. $\mathscr{Y}$ is separated, Example/Definition/Warning 2.8, so, for example, the classifier $[Y / G]$ of a finite group action on a separated manifold, then $D$ in (72) is proper.

Proof By definition, (7), $\mathscr{Y}$ is the classifier of an étale groupoid, so the diagonal is always representable and net, while proper, is an an étale local property so we can apply Proposition 1.1.

All of which may be wholly satisfactory for finite group actions, but it doesn't cover what should be a good case such as $\mathbb{R} / \mathbb{Q}$, or, more generally, Example 1.3, discrete group actions on separated manifolds, to this end, let us introduce a topological variant of the valuative criteria of properness that one encounters in algebraic geometry, to wit:

Definition A.10 Let $I^{*}$ be the semi-open interval $[0,1)$ and $I$ the closed interval $[0,1]$, then a continuous map $f: X \rightarrow Y$ of topological spaces is said to be path separated if given a commutative diagram,

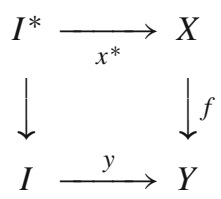

and maps $a, b: I \rightarrow X$ whose restriction to $I^{*}$ is $x^{*}$, then $a=b$. Similarly, $f$ is path proper if it is path separated, and there exists $x: I \rightarrow X$ whose restriction to $I^{*}$ is $x^{*}$. In order to fix ideas, let us make,

Remark A.11 Plainly separated, resp. proper, implies path separated, resp. proper, but the converse is certainly false, e.g. for $\Gamma$ a discrete group acting on a separated space,

$$
Y \times \Gamma \rightarrow Y \times Y:(y, \gamma) \mapsto\left(y, y^{\gamma}\right)
$$

is path proper, and, although it's a separated map, it may well fail to be proper, e.g. $\mathbb{R} / \mathbb{Q}$.

At the same time, under mild hypothesis separation and path separation of manifolds in the text book sense are the same thing, for example: 
Fact A.12 A topological space, $X$, is path separated, i.e. $X \rightarrow$ pt is path separated, iff its diagonal is path proper. Similarly if $X$ is a path separated first countable, locally path connected topological space for which around any two points $x, y$, there are decreasing sequences of open neighbourhoods,

$$
\begin{aligned}
& U_{0} \supset U_{1} \supset \cdots \supset U_{n} \ni x \quad \bigcap_{n} U_{n}=x, \quad n \in \mathbb{Z}_{>0}, \\
& V_{0} \supset V_{1} \supset \cdots \supset V_{n} \ni y \quad \bigcap_{n} V_{n}=y, \quad n \in \mathbb{Z}_{>0}
\end{aligned}
$$

affording a basis of the topology around $x$, and $y$ such that for any $n \in \mathbb{Z}_{>0}, \pi_{0}\left(U_{n} \cap V_{n}\right)$ is finite, then $X$ is separated.

Proof The first part is just the definitions. As to the second suppose a sequence $z_{m}$ limits on both $x$, and $y$, then after subsequencing and re-indexing as necessary we can suppose that for $m \geqslant n$, every $z_{m}$ is contained in the same connected component of $U_{n} \cap V_{n}$. Consequently, there is a path $\gamma_{n}$ from $z_{n}$ to $z_{n+1}$ wholly contained in $U_{n} \cap V_{n}$, and we can build a path,

$$
I^{*} \stackrel{z}{\longrightarrow} X: z_{1} \stackrel{\gamma_{1}}{\longrightarrow} z_{2} \stackrel{\gamma_{2}}{\longrightarrow} \cdots \text { etc. }
$$

which limits on both $x$, and $y$ so, by our path separation hypothesis, $x=y$.

Consequently we have a reasonable, and more flexible, alternative to proper to which we can apply the main theorem, Proposition 1.1, thanks to,

Fact A.13 Both path separated and path proper are, in the sense of Definition 2.6, étale local properties.

Proof To verify that either property is closed under base change is a diagram chase, i.e. consider the commutative diagram in which the rightmost square is fibred,

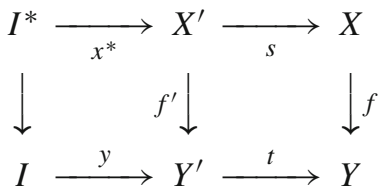

then to give $a, b: I \rightarrow X^{\prime}$ extending $x^{*}$ while lifting $y$ is equivalent to giving $s a$, $s b$ extending $s x^{*}$ alone, since necessarily $f^{\prime} a=f^{\prime} b=y$, so if $f$ is path separated $s a=s b, f^{\prime} a=f^{\prime} b$ and whence $a=b$ by the definition of fibre products. Similarly if $f$ is path proper then there is a $\xi: I \rightarrow X$ extending $s x^{*}$ such that $f \xi=t y$, and the couple $(\xi, y)$ defines the extension of $x^{*}$, again, by the universal property.

Now suppose $V \rightarrow Y$ is an étale atlas such that the fibre $X_{V} \rightarrow Y_{V}$ is path separated, respectively proper, then by the first part we can suppose that $V$ is an open cover $\bigsqcup_{\alpha} V_{\alpha}$. As such, if we choose $V_{\alpha}$ to be a neighbourhood of $y(1)$, and shrink $I$ to some $[1-\epsilon, 1]$ then we have (73) with $Y^{\prime}=V_{\alpha}$ an open embedding and $f^{\prime}$ path separated, resp. proper, whence the same for $f$.

As such we may conclude our tour of separation by way of a final, 
Example A.14 Let everything be as in Example A.9 then we may replace proper by path proper in item (b) of op. cit.. In particular, therefore, if $\mathscr{Y}=[Y / \Gamma]$ is the classifier of a discrete group action on a separated manifold, the fibre in (72) is either empty or a net path proper map from a separated manifold of co-dimension $q$.

Acknowledgements The origin of this paper is an ongoing (albeit currently stalled) attempt with Yo'av Rieck to prove an analogue of Papakyriakopoulos' sphere theorem for 3-orbifolds, and it is a pleasure to thank him for many stimulating, and informative discussions.

Funding Open access funding provided by Universitá degli Studi di Roma Tor Vergata within the CRUICARE Agreement.

Open Access This article is licensed under a Creative Commons Attribution 4.0 International License, which permits use, sharing, adaptation, distribution and reproduction in any medium or format, as long as you give appropriate credit to the original author(s) and the source, provide a link to the Creative Commons licence, and indicate if changes were made. The images or other third party material in this article are included in the article's Creative Commons licence, unless indicated otherwise in a credit line to the material. If material is not included in the article's Creative Commons licence and your intended use is not permitted by statutory regulation or exceeds the permitted use, you will need to obtain permission directly from the copyright holder. To view a copy of this licence, visit http://creativecommons.org/licenses/by/4.0/.

\section{References}

1. Behrend, K., Xu, P.: Differentiable stacks and gerbes. J. Symplectic Geom. 9(3), 285-341 (2011). URL http://projecteuclid.org/euclid.jsg/1310388899

2. Bernard, P., Mandorino, V.: Some remarks on Thom's transversality theorem. Ann. Sc. Norm. Super. Pisa Cl. Sci. 14(2), 361-386 (2015)

3. Grothendieck, A.: Éléments de géométrie algébrique. I. Le langage des schémas. Publ. Math. Inst. Hautes Études Sci. (4), 228 (1960). URL http://www.numdam.org/item?id=PMIHES_1960_4_228_ 0

4. Grothendieck, A.: Éléments de géométrie algébrique. IV. Étude locale des schémas et des morphismes de schémas IV. Publ. Math. Inst. Hautes Études Sci. (32), 361 (1967). Rédigés avec la collaboration de Jean Dieudonné

5. Grothendieck, A.: Revêtements étales et groupe fondamental (SGA 1). Lecture Notes in Mathematics, vol. 224. Springer, Berlin (1971)

6. Grothendieck, A.: Théorie des topos et cohomologie étale des schémas. Tome 1: Théorie des topos. Lecture Notes in Mathematics, vol. 269. Springer, Berlin (1972). Séminaire de Géométrie Algébrique du Bois-Marie 1963-1964 (SGA 4), Dirigé par M. Artin, A. Grothendieck, et J. L. Verdier. Avec la collaboration de N. Bourbaki, P. Deligne et B. Saint-Donat

7. Heinloth, J.: Notes on differentiable stacks. Mathematisches Institut. Georg-August-Universität Göttingen: Seminars Winter Term 2004(2005), pp. 1-32. Universitätsdrucke Göttingen, Göttingen (2005)

8. Hepworth, R.: Morse inequalities for orbifold cohomology. Algebr. Geom. Topol. 9(2), 1105-1175 (2009). https://doi.org/10.2140/agt.2009.9.1105

9. Hiltunen, S.: Implicit functions from locally convex spaces to Banach spaces. Studia Math. 134(3), 235-250 (1999). https://doi.org/10.4064/sm-134-3-235-250

10. Hirsch, M.W.: Differential Topology. Graduate Texts in Mathematics, vol. 33. Springer, New York (1994). Corrected reprint of the 1976 original

11. Laumon, G., Moret-Bailly, L.: Champs Algébriques. Ergebnisse der Mathematik und ihrer Grenzgebiete, vol. 39. Springer, Berlin (2000)

12. Madsen, I., Rothenberg, M.: On the classification of $G$-spheres. I. Equivariant transversality. Acta Math. 160(1-2), 65-104 (1988). https://doi.org/10.1007/BF02392273

13. Navarro González, J.A., Sancho de Salas, J.B.: $C^{\infty}$-Differentiable Spaces. Lecture Notes in Mathematics, vol. 1824. Springer, Berlin (2003). https://doi.org/10.1007/b13465 
14. Petrie, T.: Equivariant quasi-equivalence, transversality and normal cobordism. Proceedings of the International Congress of Mathematicians Vancouver 1, 537-541 (1974)

15. Roberts, D.M., Vozzo, R.F.: The smooth Hom-stack of an orbifold. In: 2016 MATRIX Annals. MATRIX Book Series, vol. 1, pp. 43-47. Springer, Cham (2018)

16. Smale, S.: An infinite dimensional version of Sard's theorem. Amer. J. Math. 87, 861-866 (1965). https://doi.org/10.2307/2373250

Publisher's Note Springer Nature remains neutral with regard to jurisdictional claims in published maps and institutional affiliations. 